\title{
Spectra of regular quantum graphs
}

\author{
Yu. Dabaghian, R. V. Jensen, and R. Blümel \\ Department of Physics, Wesleyan University, \\ Middletown, CT 06459-0155, USA
}

(November 2, 2018)

\begin{abstract}
We consider a class of simple quasi one-dimensional classically non-integrable systems which capture the essence of the periodic orbit structure of general hyperbolic nonintegrable dynamical systems. Their behavior is simple enough to allow a detailed investigation of both classical and quantum regimes. Despite their classical chaoticity, these systems exhibit a "nonintegrable analog" of the Einstein-Brillouin-Keller quantization formula which provides their spectra explicitly, state by state, by means of convergent periodic orbit expansions. 05.45.Mt, 03.65.Sq, 02.30.Lt
\end{abstract}

Typeset using REVTEX 


\section{INTRODUCTION}

Very few quantum systems can be solved explicitly. Among them are the standard textbook examples, such as the harmonic oscillator or the hydrogen atom [1]. In all of these cases the spectrum of the quantum system is obtained as an explicit analytical formula of the form " $E_{n}=\ldots$ ", where $n$ is the quantum number of the system. This procedure fails already for some of the simplest quantum systems, which are still considered elementary textbook problems. An example is a quantum particle in a box with a step potential inside, as shown in Fig. 1. Even for the simple problem of Fig. 1, explicit analytical solutions of the form " $E_{n}=\ldots$ " are no longer available since the problem leads to a transcendental spectral equation. The recommended method of solution is either numerical or graphical [1] 3]. We recently found a way [4] of obtaining explicit analytical solutions of a wide class of problems such as the one shown in Fig. 1, thus obtaining an explicit analytical solution of textbook problems which until now were relegated to numerical or graphical solution techniques. Our methods are also a step forward in the mathematical theory of almost periodic functions [7] since we obtain explicit formulae for the zeros of a wide class of almost periodic functions. Furthermore, the classical dynamics of the quantum systems to be discussed in this paper is chaotic. Since it may well be true in general that the quantized versions of classically chaotic systems do not admit for the existence of quantum numbers (see, e.g., [8.9] for a detailed discussion of this important point), our " $E_{n}=\ldots$ " spectral formulae, containing an explicit quantum number $n$, may come as a surprise. At this point we feel that it is important to stress that our results are not conjectures, approximations or merely formal identities. Our results are exact, explicit, convergent periodic orbit expansions that can be cast into the form of mathematical theorems. We will publish the rigorous mathematical underpinnings of our results elsewhere [10].

It is well known [11] that the periodic orbit theory leads to completely different approaches for quantizing integrable and non-integrable dynamical systems. For integrable systems there is a simple procedure [11, 12] which allows us to quantize the action vari- 
ables individually for each degree of freedom. The situation is completely different for the chaotic case, where the periodic orbit theory [11] allows us to evaluate only certain global characteristics of the spectrum, e.g. the density of states

$$
\rho(E)=\sum_{j=1}^{\infty} \delta\left(E-E_{j}\right) \approx \bar{\rho}(E)+\frac{1}{\pi} \operatorname{Im} \sum_{p} T_{p}(E) \sum_{\nu=1}^{\infty} A_{p}^{\nu}(E) e^{i \nu S_{p}(E)}
$$

typically only with semiclassical accuracy [13]. Here $\bar{\rho}(E)$ is the average density of states, $S_{p}(E), T_{p}(E)$ and $A_{p}(E)$ are correspondingly the action, the period and the weight factor of the prime periodic orbit labeled by $p$, and $\nu$ is the repetition index. In this approach individual energy levels are obtained indirectly as the singularities of the sum (1.1). As for the idea of expressing them directly in terms of the periodic orbits, M. V. Berry wrote in 1991 [14: “...We do not know how, or even whether, the closed orbit sum generates the individual $\delta$ s in the level density for chaotic systems. This is a serious - perhaps shockingsituation, because it means that we are ignorant of the mechanism of quantization".

In the case of quantum graphs Berry's question can be answered definitively. The periodic orbit sums representing the spectral density of quantum graphs do provide the individual levels in the form of $\delta$-spikes in (1.1) and only those [15 18]. In addition we showed recently [4] that the answer to Berry's question can be taken one step forward: not only do periodic orbit expansions for quantum graphs produce $\delta$-functions for the quantum states in the level density, but for certain classes of quantum graphs there also exist explicit convergent periodic orbit expansions for individual energy levels. Because they provide explicit formulas for the energy levels of classically chaotic systems, these periodic orbit expansions may be considered as "nonintegrable analogues" of the Einstein-Brillouin-Keller (EBK) quantization formula 11,12 which applies to integrable systems.

This paper is organized as follows. In Sect. II we briefly review the theory of quantum graphs and extend the theory by defining "dressed graphs", i.e. quantum graphs with arbitrary potentials on their bonds. In Sect. III we define an important class of dressed quantum graphs: regular quantum graphs. Based on a detailed study of their spectral properties in Sect. III we derive explicit analytical spectral formulae for regular quantum 
graphs in Sect. IV. In Sect. V we present a variety of regular quantum graphs illustrating use and convergence of the spectral formulae. In Sect. VI we summarize our results and conclude the paper.

\section{DYNAMICAL NETWORKS}

Let us consider a particle moving on a quasi one-dimensional network of bonds and vertices. In the mathematical literature these networks are known as graphs. They were and still are the subject of intensive investigations in all areas of science ranging from mathematics over computer science to chemistry and physics. An example of a simple graph with five vertices and seven bonds is shown in Fig. 2. The particle scatters randomly at every vertex $V_{i}$ along different bonds $B_{i j}$ which meet at that vertex. We shall assume that the graph contains a finite number of bonds and vertices ( $N_{B}$ and $N_{V}$ correspondingly). The key assumption about the dynamics of the particle is that the turning points of any trajectory of a particle moving on the graph coincide with the vertices of the graph, and hence the shape of the trajectories is determined uniquely by the geometry of the graph. The trajectories of the particle are simply the joint sequences of graph bonds, which are easily described and enumerated. For instance, every trajectory can be represented by a sequence of $N_{B}$ symbols, each one of which corresponds to a certain bond 19. Since the trajectories correspond to various bond sequences, every trajectory is described by a code word consisting of $N_{B}$ symbols.

We "dress" the bonds $B_{i j}$ of the graph with potentials, $U_{i j}(x)$, which may affect the way a particle moves along the bonds. However, it is required that these dressings do not violate the geometry of the particle trajectories, i.e. do not add turning points, other than the original vertices of the graph. This condition is required to hold at all energies. To comply with this requirement the bond potentials are allowed to depend on the energy $E$ of the particle, i.e. $U_{i j}=U_{i j}(x, E)$, such that $E>U_{i j}(x, E)$ is fulfilled for all $E$ and all $i, j$. This will in fact lead to many additional simplifications which have a deep physical meaning in 
the context of the semiclassical periodic orbit theory [6, 6, 19 21].

The shapes of the trajectories, and in particular of the periodic orbits, becomes increasingly complicated as their lengths grow. This is what makes them similar to the generic (dynamical) chaotic systems. In fact, the number of possible periodic orbits increases exponentially with their lengths, (or, equivalently, the number of vertex scatterings) with a rate which depends only on the topology of the graph. Every graph $\Gamma$ can be characterized by its topological entropy (global average rate of exponential proliferation of periodic orbits)

$$
\Lambda_{\Gamma}=\lim _{l \rightarrow \infty} \frac{\ln [\#(l)]}{l}
$$

where $l$ characterizes the lengths of the periodic orbits in terms of the lengths of their code words and $\#(l)$ is the total number of periodic orbits of length $\leq l[9]$. Since the phase space of the system is bounded, the dynamics of the particle is mixing [16]. Hence the structure of the periodic orbit set on dynamical networks closely imitates the behavior of the closed trajectories of generic chaotic systems [22,23]. On the other hand, dynamical networks can be easily quantized $44,6,17,19,24$, which makes them very convenient models for studying various aspects of quantum chaology.

The details of the classical dynamics on graphs are discussed in numerous publications 16,25]. Below we investigate the quantum-mechanical description of these systems. In particular, we shall discuss their spectra in the context of the periodic orbit theory. So let us now briefly outline some details of the graph quantization procedure, which will be used in the subsequent discussion.

A quantum graph system is a quantum particle which moves on a one-dimensional network $\Gamma$ dressed with the potentials $U_{i j}(x, E)$. Below we shall consider the case of scaling potentials discussed in [6,26, 28],

$$
U_{i j}(E)=\lambda_{i j} E, \quad \lambda_{i j}=\lambda_{j i}
$$

where the $\lambda_{i j}$ 's are constants. This choice of the dressing potentials allows us to avoid certain mathematical complications, which are irrelevant for the physical context of our discussion. 
For more details on scaling potentials and their relevance in the semiclassical periodic orbit analysis see [4,6, 19].

The Schrödinger equation for graphs with the potentials (2.2) can be written as

$$
\hat{\pi}_{i j}^{2} \psi_{i j}(x)=\beta_{i j}^{2} E \psi_{i j}(x)
$$

where

$$
\hat{\pi}_{i j}=-i \frac{d}{d x}-A_{i j}
$$

is the generalized momentum operator and $\beta_{i j}^{2}=1-\lambda_{i j}$. The coordinate $0 \leq x \leq L_{i j}$ is measured along $B_{i j}$ from $i$ to $j$, and $L_{i j}=L_{j i}$ is the length of the bond. The magnetic field vector potential $A_{i j}=-A_{j i}$ is assumed to be a constant, real matrix, and may be used as a tool for braking the time-reversal symmetry.

Classically, the particle can travel along the bond $B_{i j}$ if its energy is above the scaled potential height, $E>U_{i j}(E),\left(\lambda_{i j}<1\right)$. In this case the solution of equation (2.3) on the bond $B_{i j}$ is a combination of the free waves,

$$
\psi_{i j}(x)=a_{i j} \frac{e^{i\left(-\beta_{i j} k+A_{i j}\right) x}}{\sqrt{\beta_{i j} k}}+b_{i j} \frac{e^{i\left(\beta_{i j} k+A_{i j}\right) x}}{\sqrt{\beta_{i j} k}}
$$

where $k=\sqrt{E}$ and the factors $\left(\beta_{i j} k\right)^{-1 / 2}$ are introduced to separate the physically meaningful flux amplitudes from the coefficients $a_{i j}$ and $b_{i j}$. In the opposite case of $\lambda_{i j}>1$, the bond $B_{i j}$ carries a linear combination of tunneling solutions. Due to the scaling assumption, there is no transition between these two cases as a function of $E$. From now on we shall assume that the energy $E$ is kept above the maximal scaled potential height,

$$
\lambda_{i j}<1, \quad i, j=1, \ldots, N_{V}
$$

At every vertex $V_{i}$, the bond wave functions satisfy the boundary conditions,

$$
\begin{aligned}
\psi_{i j}(x=0) & =\varphi_{i} C_{i j}, \\
\left.\sum_{j=1}^{N_{V}} C_{i j} \hat{\pi}_{i j} \psi_{i j}(x)\right|_{x=0} & =-i \lambda_{i} \varphi_{i}
\end{aligned}
$$


for all $i, j=1, \ldots, N_{V}$. Here $C_{i j}$ is the connectivity matrix of the graph, $\varphi_{i}$ is the value of the wave function at the vertex $V_{i}$, and the $\lambda_{i}$ 's are free parameters of the problem, scaled as $\lambda_{i}=\lambda_{i}^{0} k$ (see Appendix). Note that the double-indexed scaling constants $\lambda_{i j}$ refer to the bonds, whereas the single-indexed constants $\lambda_{i}$ refer to the scattering strengths at the vertices. We believe that this notation is natural and does not lead to confusion.

The conditions (2.7) are consistent only for a discrete set of energy levels, $E_{n}=k_{n}^{2}$ which define the spectrum of the dressed quantum graph problem (2.3) and (2.7). As shown in [6,16 18,24 (see Appendix), using the scattering quantization approach [29], one obtains the spectral equation for any quantum graph problem in the form

$$
\Delta(k)=\operatorname{det}[1-S(k)]=0,
$$

where $S(k)$ is the finite unitary graph scattering matrix [16]. The indices that define the matrix elements $S_{I J}$ of the matrix $S$ correspond to the graph bonds. It is important that the bond $B_{I} \equiv B_{i j}$ is considered to be different from the (geometrically identical) reversed bond $B_{I^{\prime}} \equiv B_{j i}$, so the bonds of the graph are directed [4, 6, 16, 18]. Hence the dimensionality of the scattering matrix is $2 N_{B} \times 2 N_{B}$. It is shown in the Appendix that $S=T D(k)$, where $T$ is a constant $2 N_{B} \times 2 N_{B}$ unitary matrix and $D$ is a diagonal unitary matrix, whose matrix elements are given by

$$
D_{I J}=\delta_{I J} e^{i\left(\beta_{I} k+A_{I}\right) L_{I}}, \quad I=1, \ldots, 2 N_{B}
$$

Since $\Delta(k)$ is a complex function, it is convenient to define the spectrum via the zeros of its absolute value,

$$
|\Delta(k)|=e^{-i \Theta_{0}(k)} \Delta(k),
$$

where $\Theta_{0}(k)$ is the complex phase of $\Delta(k)$. The logarithmic derivative of $|\Delta(k)|$ produces a delta-peak for every one of its roots,

$$
-\frac{1}{\pi} \operatorname{Im} \lim _{\epsilon \rightarrow 0} \frac{d}{d k} \ln |\operatorname{det}[1-S(k+i \epsilon)]|=\sum_{n=1}^{\infty} \delta\left(k-k_{n}\right),
$$


which, by definition, is the density of the momentum states $\rho(k)$ [6]. On the other hand, using (2.10) and expanding the logarithm of the determinant (2.8), the density of states can be written as

$$
\rho(k)=\frac{1}{\pi} \frac{d \Theta_{0}(k)}{d k}+\frac{1}{\pi} \operatorname{Im} \frac{d}{d k} \sum_{n=1}^{\infty} \frac{1}{n} \operatorname{Tr}[S(k)]^{n} .
$$

Then it can be easily seen from the structure of the scattering matrix $S$ [16, 24], that the matrix elements of its $n$-th power are defined on connected sequences of $n$ bonds and the trace of $S^{n}$ generates terms defined on closed connected sequences of $n$ bonds [6, 17, 24, 25].

These periodic connected sequences of $n$ bonds $B_{i j}$, can be viewed as the periodic orbits traced by a classical point particle moving on the graph. Note that the phase of the exponent in (2.9) is exactly the action of a classical point particle's trajectory traversing the bond $B_{I}$,

$$
\mathcal{S}_{\mathcal{I}}=\int_{B_{I}}\left(\beta_{I} k+A_{I}\right) d x=\left(\beta_{I} k+A_{I}\right) L_{I}
$$

Thus the semiclassical transition amplitudes $e^{i \mathcal{S}_{I}}$ between the vertices connected by the bond $B_{I}$ determine the scattering matrix $S(k)$. As a consequence [4, 6, 16, 19, the "closed bond sequence expansion" (2.12) can be written explicitly as a periodic orbit expansion in terms of the phases (2.13),

$$
\rho(k)=\bar{\rho}(k)+\frac{1}{\pi} \operatorname{Re} \sum_{p} S_{p}^{0} \sum_{\nu=1}^{\infty} A_{p}^{\nu} e^{i \nu S_{p}^{0} k},
$$

where $S_{p}^{0}$ is the $k$-independent "action length" of the orbit $p$,

$$
S_{p}=\sum_{p} \beta_{i j} L_{i j} k \equiv S_{p}^{0} k
$$

and $A_{p}$ is its weight containing the constant factor $e^{i \sum_{p} A_{i j} L_{i j}}$. Due to the scaling assumption (see Appendix) the weight factor $A_{p}$ is $k$-independent. The first term in this expression corresponds to the average density of states of momentum $\bar{\rho}(k)$,

$$
\bar{\rho}(k)=\frac{1}{\pi} \frac{d \Theta_{0}(k)}{d k},
$$

while the periodic orbit sum in (2.14) describes the fluctuations around the average. 
The periodic orbit expansion for the staircase function

$$
N(k)=\sum_{n=1}^{\infty} \Theta\left(k-k_{n}\right)
$$

can be obtained by direct integration of (2.11) and (2.14). We obtain

$$
N(k)=\bar{N}(k)+\tilde{N}(k),
$$

where the first term,

$$
\bar{N}(k)=\int_{0}^{k} \bar{\rho}\left(k^{\prime}\right) d k^{\prime}+\bar{N}(0)
$$

represents the average behavior of the staircase and

$$
\tilde{N}(k)=\operatorname{Im} \frac{1}{\pi} \sum_{p} \sum_{\nu=1}^{\infty} \frac{A_{p}^{\nu}}{\nu} e^{i \nu S_{p}^{0} k}
$$

describes zero-mean oscillations around the average.

As discussed in the Introduction (see also [4 6, 16, 25]), quantum graphs are chaotic in the classical limit. The classical scattering probabilities are obtained in the limit $\hbar \rightarrow 0$ from the quantum mechanical transition amplitudes [46], (see Appendix). In the scaling case they are $k$-independent and thus the quantum scattering amplitudes do not depend on $\hbar$ at all. They determine simultaneously the quantum and the classical scattering probabilities.

\section{REGULAR GRAPHS AND THEIR SPECTRA}

The spectral determinant is a polynomial of degree $2 N_{B}$ of the matrix elements of $S$. It was shown in [6] that the total phase of this polynomial is

$$
\Theta_{0}(k)=\frac{1}{2} \operatorname{Im} \ln \operatorname{det} S(k)=k S_{0}-\pi \gamma_{0},
$$

where $S_{0}=\sum_{(i j)} L_{i j} \beta_{i j}$ is the total action length of the graph $\Gamma$ and

$$
\gamma_{0}=\left(N_{B}+N_{V}\right) / 2+\frac{1}{\pi} \sum_{i=1}^{N_{V}} \arctan \left(\frac{\lambda_{i}^{0}}{v_{i}}\right),
$$

where 


$$
v_{i}=\sum_{j} C_{i j} \beta_{i j}
$$

Therefore the average density of states is a constant,

$$
\bar{\rho}=\frac{1}{\pi} \frac{d}{d k} \Theta_{0}(k)=\frac{S_{0}}{\pi}
$$

and the average staircase (2.19) is

$$
\bar{N}(k)=\frac{S_{0}}{\pi} k+\bar{N}(0)
$$

The spectral equation $|\Delta(k)|=0$ can be written in the form

$$
\cos \left(S_{0} k-\pi \gamma_{0}\right)=\sum_{i=1}^{N_{\Gamma}} a_{i} \cos \left(S_{i} k-\pi \gamma_{i}\right)
$$

where the frequencies $S_{i}<S_{0}$ are combinations of the reduced classical actions $S_{i j}^{0}=\beta_{i j} L_{i j}$, and $\gamma_{0}, \gamma_{i}$ are constants. The number $N_{\Gamma}$ of terms in (3.6) is bounded by $N_{\Gamma} \leq 3^{N_{B}}$ [6].

The frequency $S_{0}$ in $\Theta_{0}(k)$ is the largest frequency in the expansion (3.6). While it is the only characteristic of the graph contained in the left-hand side of (3.6), the right-hand side

$$
\Phi(k) \equiv \sum_{i=1}^{N_{\Gamma}} a_{i} \cos \left(S_{i} k-\pi \gamma_{i}\right),
$$

contains the complete information about the graph system. We call $\Phi(k)$ the characteristic function of the graph.

A graph $\Gamma$ is called regular [4], if its characteristic function $\Phi(k)$ satisfies

$$
\sum_{i=1}^{N_{\Gamma}}\left|a_{i}\right| \equiv \alpha<1
$$

For regular graphs the spectral equation (3.6) can be solved formally [4 to yield the implicit equation of its eigenvalues,

$$
k_{n}=\frac{\pi}{S_{0}}\left[n+\mu+\gamma_{0}\right]+\frac{1}{S_{0}} \begin{cases}\arccos \left[\Phi\left(k_{n}\right)\right], & \text { for } n+\mu \text { even } \\ \pi-\arccos \left[\Phi\left(k_{n}\right)\right], & \text { for } n+\mu \text { odd }\end{cases}
$$

where $\mu$ is a fixed integer, chosen such that $k_{1}$ is the first positive solution of (3.6). The index $n \in \mathbf{N}$ labels the roots of (3.6) in their natural sequence. 
From the implicit form (3.9) it follows immediately that since the second term in (3.9) is bounded by $\pi / S_{0}$, the deviations of solutions to this equation from the points

$$
\hat{k}_{n}=\frac{\pi}{S_{0}}\left(n+\mu+\gamma_{0}+1\right)
$$

never exceeds $\pi / S_{0}$ in absolute value for any $n$. Below it will turn out that the quantities $\hat{k}_{n}$ are very important since they determine the root structure of (3.6).

The roots $k_{n}$ can be decomposed into an average part $\bar{k}_{n}$ and a fluctuating part $\tilde{k}_{n}$. From (3.9) we obtain

$$
k_{n}=\bar{k}_{n}+\tilde{k}_{n}
$$

where

$$
\bar{k}_{n}=\frac{\pi}{S_{0}}\left[n+\mu+\gamma_{0}+1 / 2\right]
$$

and

$$
\tilde{k}_{n}=\frac{(-1)^{n+\mu}}{S_{0}}\left\{\arccos \left[\Phi\left(k_{n}\right)\right]-\frac{\pi}{2}\right\}
$$

Note that the constant $\mu+\gamma_{0}$ can be related to the initial value $\bar{N}(0)$ of the average staircase function (3.5). Consider the integral

$$
\lim _{n \rightarrow \infty} \frac{1}{\hat{k}_{n}} \int_{0}^{\hat{k}_{n}} N\left(k^{\prime}\right) d k^{\prime}
$$

The integration in (3.14) can be easily performed due to the simple form (2.17) of the function $N(k)$ :

$$
\frac{1}{\hat{k}_{n}} \int_{0}^{\hat{k}_{n}} N\left(k^{\prime}\right) d k^{\prime}=n-\frac{1}{\hat{k}_{n}} \sum_{i=1}^{n} k_{i},
$$

since there are $n$ roots to the left of $\hat{k}_{n}$. The fluctuations of both $N(k)$ and $k_{n}$ around their averages have zero mean, so in the limit of $n \gg 1$ one can use $\bar{N}(k)$ and $\bar{k}_{n}$ instead of $N(k)$ and $k_{n}$ in (3.15), and write

$$
\frac{1}{\hat{k}_{n}} \int_{0}^{\hat{k}_{n}} \bar{N}\left(k^{\prime}\right) d k^{\prime}=n-\frac{1}{\hat{k}_{n}} \sum_{i=1}^{n} \bar{k}_{i}, \quad n \gg 1 .
$$


Using the explicit forms of $\hat{k}_{n}, \bar{k}_{n}$ and $\bar{N}(k)$, we obtain

$$
\bar{N}(0)+\frac{1}{2}\left(n+\mu+\gamma_{0}+1\right)=\frac{n}{n+\mu+\gamma_{0}+1}\left(\frac{n}{2}+\mu+\gamma_{0}+1\right) .
$$

Expanding the right-hand side and keeping terms up to order $1 / n$ yields

$$
\bar{N}(0)+\frac{1}{2}\left(n+\mu+\gamma_{0}+1\right)=n-\left(1-\frac{\mu+\gamma_{0}+1}{n}\right)\left(\frac{n}{2}+\mu+\gamma_{0}+1\right) .
$$

The terms proportional to $n$ cancel out. Comparing the constants in (3.18) yields

$$
\bar{N}(0)=-\left(\mu+\gamma_{0}+1\right)
$$

One can verify by direct substitution that

$$
\bar{N}\left(\hat{k}_{n}\right)=n
$$

which implies that the function $(3.10)$ is the inverse of the average staircase (3.5). One can also view the points $\hat{k}_{n}$ as the intersection points of the staircase (2.17) and its average (3.5),

$$
\bar{N}\left(\hat{k}_{n}\right)=N\left(\hat{k}_{n}\right)=n
$$

so at the points $\hat{k}_{n}$ the fluctuations $\tilde{N}(k)$ of the spectral staircase vanish,

$$
\tilde{N}\left(\hat{k}_{n}\right)=\operatorname{Im} \frac{1}{\pi} \sum_{p} \sum_{\nu=1}^{\infty} \frac{A_{p}^{\nu}}{\nu} e^{i \nu S_{p}^{0} \hat{k}_{n}}=0 .
$$

Geometrically, (3.22) means that the average staircase $\bar{N}(k)$ intersects every step of the staircase function $N(k)$. Hence we call $\bar{N}(k)$ the piercing average. This is illustrated in Fig. 3 which shows the spectral staircase $N(k)$ for the scaling step potential shown in Fig. 1 and discussed in more detail in Sect. V, Example 1, below. We used the parameters $\lambda=1 / 2$ and $b=0.3$. Also shown is the average staircase $\bar{N}(k)$ for this case. It clearly pierces all the steps of $N(k)$ providing an example of a system with a piercing average.

Since $\Phi(k)$ contains only frequencies smaller than $S_{0}$, every open interval $I_{n}=\left(\hat{k}_{n-1}, \hat{k}_{n}\right)$ contains only one root of (3.6), namely $k_{n}$. Thus the $\hat{k}_{n}$ play the role of separating points between adjacent roots. [ㅂ 6, 10]. Moreover, because of (3.8), the "allowed zones" $R_{n} \subset I_{n}$, where the roots $k_{n}$ can be found, narrow to 


$$
k_{n} \in R_{n} \equiv\left(\frac{\pi}{S_{0}}\left(n+\mu+\gamma_{0}+u\right), \frac{\pi}{S_{0}}\left(n+\mu+\gamma_{0}+1-u\right)\right)
$$

where $u=\arccos (\alpha) / S_{0}$. Correspondingly, there are forbidden regions $F_{n}$,

$$
F_{n} \equiv\left(\frac{\pi}{S_{0}}\left(n+\mu+\gamma_{0}-u\right), \frac{\pi}{S_{0}}\left(n+\mu+\gamma_{0}+1+u\right)\right)
$$

where roots of (3.6) never appear. In the limit $\alpha \rightarrow 1(u \rightarrow 0)$ the allowed zones $R_{n}$ tend to occupy the whole root interval, $R_{n} \rightarrow I_{n}$.

\section{SPECTRAL FORMULAE}

Once the existence of separating points $\hat{k}_{n}$ has been established, it is possible to obtain an exact periodic orbit expansion separately for every root of (2.8). The derivation is based on the identity

$$
k_{n}=\int_{\hat{k}_{n-1}}^{\hat{k}_{n}} k \rho(k) d k .
$$

Substituting the exact periodic orbit expansion (2.14) for $\rho(k)$ into (4.1), yields

$$
\begin{array}{r}
k_{n}=\int_{\hat{k}_{n-1}}^{\hat{k}_{n}} k \frac{S_{0}}{\pi} d k+\frac{1}{\pi} \int_{\hat{k}_{n-1}}^{\hat{k}_{n}} k \operatorname{Re} \sum_{p} S_{p}^{0} \sum_{\nu=1}^{\infty} A_{p}^{\nu} e^{i \nu S_{p}^{0} k} d k=\frac{\pi}{S_{0}}\left(n+\mu+\gamma_{0}+1 / 2\right) \\
+\hat{k}_{n} \operatorname{Im} \frac{1}{\pi} \sum_{p} \sum_{\nu=1}^{\infty} \frac{A_{p}^{\nu}}{\nu} e^{i \nu S_{p}^{0} \hat{k}_{n}}-\hat{k}_{n-1} \operatorname{Im} \frac{1}{\pi} \sum_{p} \sum_{\nu=1}^{\infty} \frac{A_{p}^{\nu}}{\nu} e^{i \nu S_{p}^{0} \hat{k}_{n-1}} \\
+\operatorname{Re} \frac{1}{\pi} \sum_{p} \frac{1}{S_{p}^{0}} \sum_{\nu=1}^{\infty} \frac{A_{p}^{\nu}}{\nu^{2}}\left(e^{i \nu S_{p}^{0} \hat{k}_{n}}-e^{i \nu S_{p}^{0} \hat{k}_{n-1}}\right) .
\end{array}
$$

Using (3.22) we simplify (4.2) to obtain

$$
k_{n}=\frac{\pi}{S_{0}}\left(n+\mu+\gamma_{0}+1 / 2\right)-\frac{1}{\pi} \operatorname{Im} \sum_{p} \frac{2}{S_{p}^{0}} \sum_{\nu=1}^{\infty} \frac{A_{p}^{\nu}}{\nu^{2}} \sin \left[\frac{\nu \omega_{p}}{2}\right] e^{i \nu \omega_{p}\left(n+\mu+\gamma_{0}+1 / 2\right)},
$$

where $\omega_{p}=\pi S_{p}^{0} / S_{0}$. The series expansion (4.3) for $k_{n}$ is more than a formal identity. It is rigorously convergent, however it converges only conditionally, which means that the result of the summation depends essentially on how the summation was performed. Indeed, according to Riemann's well-known reordering theorem, one can obtain any result by rearranging the terms of a conditionally convergent series [30]. Hence for proper convergence of (4.3) to the 
exact roots of the spectral equation (3.6), we have to specify how the terms in (4.3) are to be summed.

The mathematical details of the convergence properties of (4.3) are presented in [10]. Here we mention the main result, which states that the terms in (4.3) have to be summed according to the length of the symbolic codes [6.,19] of the periodic orbits, and not according to their action lengths. If (4.3) is summed in this way it not only converges, but also converges to the exact roots $k_{n}$ of the spectral equation (3.6).

Hence the formula (4.3) provides an explicit representation of the roots of the spectral equation (2.8) in terms of the geometric characteristics of the graph. According to (3.12), the first term in (4.3) is the average $\bar{k}_{n}$, and the following periodic orbit sum is an explicit expression for the fluctuation of the root $\tilde{k}_{n}$. This method is not limited to obtaining explicit analytical periodic orbit expansions for $k_{n}$. In fact, by using the identity

$$
f\left(k_{n}\right)=\int_{\hat{k}_{n-1}}^{\hat{k}_{n}} f(k) \rho(k) d k,
$$

we can obtain periodic orbit expansions for any function of the eigenvalues $f\left(k_{n}\right)$, for instance for the energy $E=k^{2}$.

In the simplest case where $\lambda_{i}^{0}=0, A_{i j}=0$ and $\operatorname{Im} A_{p}=0$ we have

$$
k_{n}=\frac{\pi}{S_{0}} n-\frac{2}{\pi} \sum_{p} \frac{1}{S_{p}^{0}} \sum_{\nu=1}^{\infty} \frac{A_{p}^{\nu}}{\nu^{2}} \sin \left(\frac{1}{2} \nu \omega_{p}\right) \sin \left(\nu \omega_{p} n\right) .
$$

Note that in this case $k_{-n}=-k_{n}$.

Both EBK theory as well as formula (4.3) allow us to compute energy eigenvalues explicitly. In this sense formula (4.3) may be regarded as an analog of the EBK quantization formula 11.12 for a chaotic system. The complexity of this expansion, structurally similar to (1.1), reflects the geometrical complexity of the periodic orbit set for graph systems.

Finally, for explicit calculations (see the following Sect. V) it remains to determine the explicit form of the expansion coefficients $A_{p}$. For some simple graphs this was done in 16,19. In the Appendix we solve the problem for general dressed graphs. We show that every passage of an orbit $p$ from a bond $B_{i j}$ to $B_{i j^{\prime}}$ through a vertex $V_{i}$ contributes a factor $\sigma_{j i, i j^{\prime}}$ (a matrix element of the matrix $T$, see Appendix) to the weight $A_{p}$ of the orbit, 


$$
A_{p}=\prod \sigma_{j j^{\prime}}^{(i)}
$$

where the product is taken over the sequence of the bonds traced by the orbit $p$.

\section{EXAMPLES}

In (3.8) we provided a definition of regular quantum graphs and discussed analytical properties of their spectra in (3.9)-(3.24). The discussion of regular quantum graphs culminated in Sect. IV with the derivation of explicit spectral formulae for individual quantum states of regular quantum graphs. It is one thing to define regular quantum graphs, and it is quite another to show that regular quantum graphs actually exist. Examples 1-3, discussed below, provide specific examples of quantum graphs that are regular for all choices of their parameters. Examples 4 and 5 present quantum graphs that exhibit both regular and irregular regimes. Finally, examples 6 and 7 provide illustrations of a new class of quantum

graphs, marginal quantum graphs, for which $\sum_{i=1}^{N_{\Gamma}}\left|a_{i}\right|=1$. Except for special choices of their dressing potentials these graphs can still be accommodated within the mathematical framework set up in Sections III and IV, and also admit an explicit representation of their spectra according to the spectral formulae derived in Sect. IV.

Example 1. Scaling step potential inside of a box. Let us consider the case of a particle confined to a box $0<x<1$ containing the scaling step potential (see Fig. 1 and Fig. 4a),

$$
U(x)= \begin{cases}0, & \text { for } 0<x \leq b, \\ \lambda_{23} E, & \text { for } b<x<1 .\end{cases}
$$

This is equivalent to a three-vertex linear chain graph (Fig. 4a'), with $\lambda_{2}=0, A_{i j}=0$ and Dirichlet boundary conditions at $V_{1}$ and $V_{3}$. This example is also discussed in [4 6, 19, 31]. In this case the spectral equation (3.6) can be written as 4,5

$$
\sin \left[k\left(S_{21}^{0}+S_{23}^{0}\right)\right]=r \sin \left[k\left(S_{21}^{0}-S_{23}^{0}\right)\right]
$$

where

$$
r=\frac{1-\beta_{23}}{1+\beta_{23}}<1
$$


is the reflection coefficient at the vertex $V_{2}$. Hence the regularity condition (3.8) is automatically satisfied and this graph is always regular. In Sect. IV we already discussed the convergence properties of (4.3), including the fact that a rigorous mathematical proof for the convergence of (4.3) exists [10]. Here we present solid numerical evidence for the convergence of (4.3) in the context of the scaling step-potential (5.1). As discussed in [4 6, 19], every periodic orbit in the potential (5.1) can be described by a binary code word. Figure 5 shows the relative error $\epsilon_{n}^{(l)}=\left|k_{n}^{(l)}-k_{n}\right| / k_{n}, n=1,10,100$, of the result $k_{n}^{(l)}$ predicted by $(4.3$ ) compared with the numerically obtained exact result $k_{n}$ as a function of the binary code length $l$ of the orbits used in the expansion (4.3). We used $b=0.3, \lambda=1 / 2$. Figure 5 also demonstrates that using all periodic orbits up to binary code length $l \sim 150$, we obtain an accuracy on the order of $10^{-4}-10^{-7}$ for the roots $k_{n}$ of (5.2). Although the convergence of the series is slow (according to Fig. 5 it is approximately on the order of $\sim 1 / l^{2}$ on average), one can obtain a fairly good estimate for the roots using all orbits of code length 20 and smaller.

Example 2. Scaling $\delta$-function in a box. This potential, shown in Fig. 4b, is again equivalent to a three-vertex quantum graph. This time, however, the potentials on the bonds are identically zero, whereas the vertex $V_{2}$ is dressed with a scaling $\delta$-function of strength $\lambda_{2}=\lambda_{2}^{0} k>0$. At the open ends we apply Dirichlet boundary conditions. In this case the spectral equation (3.6) is

$$
\cos \left[k\left(S_{21}^{0}+S_{23}^{0}\right)-\pi \gamma_{0}\right]=-|r| \cos \left[k\left(S_{21}^{0}-S_{23}^{0}\right)\right]
$$

where

$$
\gamma_{0}=1-\frac{1}{\pi} \arcsin \left(\frac{2}{\sqrt{4+\left(\lambda_{2}^{0}\right)^{2}}}\right)
$$

and the reflection coefficient $r$ is given by

$$
r=\frac{\lambda_{2}^{0}}{2 i-\lambda_{2}^{0}} .
$$

Because of $|r|<1$, the characteristic function of (5.4) also satisfies the regularity condition (3.8). Therefore the scaling $\delta$ function in a box is another example of a regular quantum 
graph.

Example 3. Combined scaling step and scaling $\delta$-potential in a box (Fig. 4c). This is equivalent to a three-vertex dressed linear graph (Fig. 4c'), with $\lambda_{2}=\lambda_{2}^{0} k>0$. The spectral equation (3.6) is

$$
\cos \left[k\left(S_{21}^{0}+S_{23}^{0}\right)-\pi \gamma_{0}\right]=a_{1} \cos \left[k\left(S_{21}^{0}-S_{23}^{0}\right)-\pi \gamma_{1}\right]
$$

where

$$
\begin{aligned}
& \gamma_{0}=1-\frac{1}{\pi} \arcsin \left(\frac{\beta_{12}+\beta_{23}}{\sqrt{\left(\beta_{12}+\beta_{23}\right)^{2}+\left(\lambda_{2}^{0}\right)^{2}}}\right) \\
& \gamma_{1}=1-\frac{1}{\pi} \arcsin \left(\frac{\beta_{12}-\beta_{23}}{\sqrt{\left(\beta_{12}-\beta_{23}\right)^{2}+\left(\lambda_{2}^{0}\right)^{2}}}\right)
\end{aligned}
$$

and the coefficient $a_{1}$ is

$$
a_{1}=\sqrt{\frac{\left(\beta_{21}-\beta_{23}\right)^{2}+\left(\lambda_{2}^{0}\right)^{2}}{\left(\beta_{21}+\beta_{23}\right)^{2}+\left(\lambda_{2}^{0}\right)^{2}}}<1 .
$$

So the characteristic function of (5.7) once again satisfies the regularity condition for any linear three-vertex graph with nontrivial bond potentials $\left(\beta_{21}^{2}+\beta_{23}^{2} \neq 0\right)[6]$.

Quantum graphs which are regular for all of their parameter values are quite exceptional. In general quantum graphs may have a regular regime for a certain range of the parameter values or the regular regime may not exist at all. The following Example 4 illustrates this point.

Example 4. Two scaling steps in a box (Fig. 4d). As an example of a graph which has both a regular and an irregular regime, let us consider a quantum particle in a box with two scaling steps (Fig. 4d), equivalent to the four-vertex linear graph shown in Fig. 4d'. Since there are no $\delta$-functions present we have $\lambda_{2}=\lambda_{3}=0$. We assume Dirichlet boundary conditions at the dead ends of this graph. In this case the spectral equation (3.6) is given by

$$
\sin \left(S_{0} k\right)=-r_{2} \sin \left(k S_{1}\right)-r_{2} r_{3} \sin \left(k S_{2}\right)+r_{3} \sin \left(k S_{3}\right)
$$

where 


$$
\begin{aligned}
& S_{0}=S_{21}^{0}+S_{23}^{0}+S_{34}^{0}, \quad S_{1}=S_{23}^{0}+S_{34}^{0}-S_{21}^{0}, \\
& S_{2}=S_{21}^{0}+S_{34}^{0}-S_{23}^{0}, \quad S_{3}=S_{21}^{0}+S_{23}^{0}-S_{34}^{0}
\end{aligned}
$$

and $r_{2}, r_{3}$ are the reflection coefficients

$$
r_{2}=\frac{\beta_{12}-\beta_{23}}{\beta_{12}+\beta_{23}}, \quad r_{3}=\frac{\beta_{23}-\beta_{34}}{\beta_{23}+\beta_{34}}
$$

at the corresponding vertices $V_{i}$. For

$$
\left|r_{3}\right|+\left|r_{2} r_{3}\right|+\left|r_{2}\right|<1
$$

the four-vertex linear graph (Fig. 4d', Fig. 6a) is regular. The regularity condition (5.13) is fulfilled in a diamond-shaped region of $\left(r_{2}, r_{3}\right)$ parameter space shown as the shaded area in Fig. 6b. The difference between the regular and the irregular regimes is clearly reflected in the staircase functions. Figure 7 a shows the staircase function $N(k)$ together with the average staircase $\bar{N}(k)$ in the regular regime for the parameter combination $r_{1}=0.2$ and $r_{3}=0.3$. The piercing-average condition is clearly satisfied. Figure $7 \mathrm{~b}$ shows the staircase function $N(k)$ together with the average staircase $\bar{N}(k)$ in the irregular regime for the parameter combination $r_{1}=0.98$ and $r_{3}=0.99$. In this case the piercing-average condition is clearly violated, consistent with the irregular nature of this regime.

Example 5. Two scaling $\delta$-functions in a box (Fig. 4e). This potential is equivalent with the four-vertex graph shown in Fig. 4e' with $\lambda_{2}=\lambda_{2}^{0} k>0, \lambda_{3}=\lambda_{3}^{0} k>0$, and Dirichlet boundary conditions at the dead ends $V_{1}$ and $V_{4}$. In this case the spectral equation (3.6) is given by

$$
\cos \left(k S_{0}-\pi \gamma_{0}\right)=a_{1} \cos \left(k S_{1}-\pi \gamma_{1}\right)+a_{2} \cos \left(k S_{2}-\pi \gamma_{2}\right)+a_{3} \cos \left(k S_{3}-\pi \gamma_{3}\right),
$$

where

$$
a_{1}=\frac{\lambda_{2}^{0}}{\sqrt{4+\left(\lambda_{2}^{0}\right)^{2}}}, \quad a_{2}=\frac{\lambda_{3}^{0}}{\sqrt{4+\left(\lambda_{3}^{0}\right)^{2}}}, \quad a_{3}=\frac{\lambda_{2}^{0} \lambda_{3}^{0}}{\sqrt{\left[4+\left(\lambda_{2}^{0}\right)^{2}\right]\left[4+\left(\lambda_{3}^{0}\right)^{2}\right]}}
$$

and 


$$
\begin{array}{lr}
\gamma_{0}=\frac{1}{\pi} \arcsin \left(\frac{\lambda_{2}^{0} \lambda_{3}^{0}-4}{\sqrt{\left[4+\left(\lambda_{2}^{0}\right)^{2}\right]\left[4+\left(\lambda_{3}^{0}\right)^{2}\right]}}\right), \\
\gamma_{1} \quad=\frac{1}{\pi} \arcsin \left(a_{2}\right), \\
\gamma_{2} & =\frac{1}{\pi} \arcsin \left(a_{1}\right), \\
\gamma_{3} & =\frac{1}{2} .
\end{array}
$$

The sum of the amplitudes in (5.15) ranges between 0 and 3. Therefore this system has regular and irregular regimes. The regular regime corresponds to a finite area in the $\left(\lambda_{2}^{0}, \lambda_{3}^{0}\right)$ parameter space. All linear chain graphs with a finite number of vertices and Dirichlet boundary conditions at the two dead-end vertices at the beginning and at the end of the graph have a finite-measure regular regime and an irregular regime. This fact is proved in 10".

A new type of graphs are marginal quantum graphs. A marginal quantum graph is defined by

$$
\sum_{i=1}^{N_{\Gamma}}\left|a_{i}\right|=1 .
$$

For marginal quantum graphs, apart from a small set of "special" graphs, explicit spectral formulae still exist. Explicit examples are provided by circular graphs (see Example 6) and star graphs (see Example 7).

Example 6. Scaling step potential in a box with periodic boundary conditions. This system is identical with the two-vertex circular graph shown in Fig. 8. In the case of a circular graph a minor notational problem arises since starting from vertex $V_{1}$, say, vertex $V_{2}$ may be reached directly via two different bonds. For the purposes of this example we solve the problem in the following way. First we introduce a positive sense of rotation, i.e. mathematically positive or counterclockwise, for the circular graph in Fig. 8. Then we introduce the scaling coefficient $\beta_{12}$ referring the the bond that connects vertex $V_{1}$ with vertex $V_{2}$ traversing the graph in the mathematically positive sense. We introduce the scaling coefficient $\beta_{21}$ which refers to the bond that connects vertex $V_{2}$ with vertex $V_{1}$, again in the mathematically positive sense. We use the same notation for the two reduced actions $S_{12}^{0}$ and 
$S_{21}^{0}$ referring to the two different bonds (in the mathematically positive sense), respectively. This notation is not confusing here, since no magnetic field is switched on $\left(A_{i j}=0\right)$. With this notation the spectral equation is given by

$$
\cos \left(k S_{0}\right)=a_{1}+a_{2} \cos \left(k S_{2}\right)
$$

where $S_{0}=S_{12}^{0}+S_{21}^{0}, S_{1}=S_{12}^{0}+S_{21}^{0}$,

$$
a_{1}=\frac{4 \beta_{12} \beta_{21}}{\left(\beta_{12}+\beta_{21}\right)^{2}}
$$

and

$$
a_{2}=\left(\frac{\beta_{12}-\beta_{21}}{\beta_{12}+\beta_{21}}\right)^{2} .
$$

Note that $a_{1}+a_{2}=1$. Condition (5.17) is satisfied and the circular quantum graph with a scaling step potential is marginal.

Although the strict inequality (3.8) is violated, it is important to note that even in the marginal case the separating points $\hat{k}_{n}$, generically, are still not solutions to (5.18). This happens only for special parameter combinations, and therefore for special quantum graphs for which the equation

$$
(-1)^{n+\mu+1}=a_{1}+a_{2} \cos \left(\hat{k}_{n} S_{2}\right)
$$

is exactly satisfied for some $n$. Since the sequence $\hat{k}_{n}$ is countable, and (5.21), in general, involves irrational requency ratios and irrational coefficients, equation (5.21) is only accidentially satisfied for some $n$ for a measure zero set of graph parameters. Hence, in general, even for marginal quantum graphs, the points $\hat{k}_{n}$ still serve as separating points and the roots of the spectral equation can still be obtained via the expansion (4.3).

Example 7. Star graph. Another example of a marginal quantum graph is provided by the star-graph shown in Fig. 9. We consider the case with three different scaling potentials on its three bonds and Dirichlet boundary conditions at the three dead ends. The spectral equation is given by 


$$
\cos \left(S_{0} k\right)=a_{1} \cos \left(S_{1} k\right)+a_{2} \cos \left(S_{2} k\right)+a_{3} \cos \left(S_{3} k\right)
$$

where

$$
\begin{aligned}
& S_{0}=S_{14}^{0}+S_{24}^{0}+S_{34}^{0}, \quad S_{1}=S_{14}^{0}-S_{24}^{0}+S_{34}^{0}, \\
& S_{2}=S_{14}^{0}-S_{24}^{0}-S_{34}^{0}, \quad S_{3}=S_{14}^{0}+S_{24}^{0}-S_{34}^{0}
\end{aligned}
$$

and

$$
a_{1}=\frac{\beta_{14}-\beta_{24}+\beta_{34}}{\beta_{14}+\beta_{24}+\beta_{34}}, \quad a_{2}=\frac{-\beta_{14}+\beta_{24}+\beta_{34}}{\beta_{14}+\beta_{24}+\beta_{34}}, \quad a_{3}=\frac{\beta_{14}+\beta_{24}-\beta_{34}}{\beta_{14}+\beta_{24}+\beta_{34}} .
$$

It is straightforward to verify that no matter what the sign of the individual $a_{i}$ in (5.24), $\sum_{i=1}^{3}\left|a_{i}\right|=1$. Therefore the condition (5.17) is satisfied and the star-graph shown in Fig. 9 is another example of a marginal quantum graph. As discussed in the context of Example 6 , and with the exception of a set of measure zero of the star-graph's parameter space, the spectral expansion (4.3) is still valid and can be used to obtain every single one of the star-graph's eigenvalues individually, and independently of all the other eigenvalues.

\section{SUMMARY, DISCUSSION AND CONCLUSION}

Exact periodic orbit expansions for the global density of states are known for many chaotic systems 15, 32, 33. However, formula $(4.3)$ is the first example of an explicit expression for the individual quantum mechanical levels, obtained as a function of the level index $n$, for a classically chaotic system. Additional explicit quantization formulas may be found for other quantum graph systems, or even for quantum systems unrelated to quantum graphs as long as two essential requirements are fulfilled. First an exact periodic orbit expansion

for the density of states must exist. Second it must be determined that one of the system's levels, $k_{*}$, is the only one in an interval $\hat{k}_{*}^{\prime}<k_{*}<\hat{k}_{*}^{\prime \prime}$. Then one can always obtain the corresponding periodic orbit expansion for $k_{*}$,

$$
k_{*}=\int_{\hat{k}_{*}^{\prime}}^{\hat{k}_{*}^{\prime \prime}} k \rho(k) d k,
$$


based on the periodic orbit expansion for $\rho(k)$.

It is reasonable to expect that generically there exist separating points $\hat{k}_{*}^{\prime}$ and $\hat{k}_{*}^{\prime \prime}$ which separate every $k_{*}$ from its neighbors, so that $k_{*}$ is the only root of the spectral equation in the interval $\left[\hat{k}_{*}^{\prime}, \hat{k}_{*}^{\prime \prime}\right]$. Hence expansions like (4.3) generally do exist. However, knowing the positions of the separators $k_{*}^{\prime}$ and $k_{*}^{\prime \prime}$ around a particular level $k_{*}$, does not help for finding the separators for all the other levels. So the most important task for obtaining a general expression for all the levels of a quantum chaotic system is finding a global function for the separating points similar to (3.10), which naturally enumerates the separators. Therefore, even though it might be possible to find, for some systems, the separators for a particular quantum level $k_{*}$, and then to obtain a periodic orbit expansion for it according to (6.1), the expansion will work only for level $k_{*}$ and will not represent a formula which can be used for obtaining other levels.

The problem of finding a global expression for the separating points as a function of their ordering index $n$ is directly related to another well-known problem of spectral theory of differential operators, namely the problem of approximating the staircase function (2.17) by a smooth average $\bar{N}(k)$. Indeed, suppose there exists a separating point $\hat{k}_{n}^{\prime}$ between every two roots of the spectral equation, i.e. a solution of the equation

$$
\bar{N}\left(\hat{k}^{\prime}\right)=N(k)
$$

between every two roots of the spectral equation (similar to (3.20) and (3.21)). Since $\bar{N}(k)$ is a monotonic function, the separating points can then be found by inverting the equation (6.2),

$$
\hat{k}_{N}=k(N)
$$

in which the value of the staircase function plays the role of the separator index $\hat{k}_{N}$. The equation (6.3) generalizes (3.10), which can be used in (6.1) to obtain the periodic orbit expansions for all of the roots.

The smooth curve defined by (6.3) where $N$ is considered to be a continuous variable, 
intersects every stair of the spectral staircase (2.17). Unfortunately, finding a smooth function which approximates the spectral staircase for a general differential operator with generic boundary conditions is a rather complicated task. It was proven by Weyl in 1912 that one can approximate $\bar{N}(k)$ by the phase-space volume of the system in question,

$$
\bar{N}(E) \approx \int \Theta(E-H(x, p)) \frac{d^{D} x d^{D} p}{(2 \pi \hbar)^{D}}
$$

where $D$ is the dimensionality of the phase space, however this average is certainly not guaranteed to satisfy the "piercing average" condition (6.2). Since Weyl, this problem has been addressed by numerous researchers (see, e.g., [34]), who succeeded in giving many improved estimates, for $\bar{N}(k)$, but none of them a priori satisfy (6.2).

The important feature of the regular quantum graph systems is that there exists a global piercing average (3.10), which uniformly enumerates all the points separating one root from another, and hence it is possible to obtain formula (4.3) as a function of the index $n$. In other words, the index $n$ in (4.3) is a quantum number, and so the expression (4.3) for the energy levels of a chaotic system in terms of classical periodic orbits can be considered as a non-integrable analog of the EBK quantization scheme [11, 12].

It should be mentioned that despite the existence of a quantum number $n$ in (4.3), the actual dependence of the energy levels on the value of its quantum number is quite different from the simple EBK scheme for integrable systems. The expansion of the fluctuating part of the roots (3.11) involves an intricate, conditionally convergent series and is rather "chaotic". The difference in complexity of the formulas (4.3) and the EBK formula apparently reflects the complexity of the geometry of the periodic orbits of the classically chaotic quantum graphs.

Y.D. and R.B. gratefully acknowledge financial support by NSF grants PHY-9900730 and PHY-9984075; Y.D. and R.V.J by NSF grant PHY-9900746. 


\section{APPENDIX}

For the sake of completeness, we present below a simple derivation of the spectral determinant (2.8), starting from the boundary conditions at the vertex $V_{i}$ :

$$
\left.\psi_{i j}(x)\right|_{x=0}=\varphi_{i} C_{i j}
$$

and

$$
\left.\sum_{j=1}^{N_{V}} C_{i j}\left(i \frac{d}{d x_{i j}}+A_{i j}\right) \psi_{i j}\left(x_{i j}\right)\right|_{x=0}=\lambda_{i} \varphi_{i}
$$

We would like to present the wave function,

$$
\psi_{i j}(x)=\frac{1}{\sqrt{\beta_{i j} k}}\left(a_{i j} e^{-i \beta_{i j} k x}+b_{i j} e^{i \beta_{i j} k x}\right),
$$

which satisfies these boundary conditions as a superposition of the partial waves

$$
\psi_{j j^{\prime}}^{(i)}\left(x_{j}\right)=\delta_{j j^{\prime}} \frac{e^{i\left(-\beta_{i j} k+A_{i j}\right) x_{j}}}{\sqrt{\beta_{i j} k}}+\sigma_{j i, i j^{\prime}} \frac{e^{i\left(\beta_{i j} k+A_{i j}\right) x_{j}}}{\sqrt{\beta_{i j} k}} .
$$

scattering on the vertices of the graph. So

$$
\psi_{i j}\left(x_{j}\right)=\sum_{j^{\prime}=1}^{N_{V}} a_{i j^{\prime}} \psi_{j, j^{\prime}}^{(i)}\left(x_{j}\right)=\frac{a_{i j}}{\sqrt{\beta_{i j} k}} e^{-i\left(\beta_{i j} k-A_{i, j}\right) x_{j}}+\frac{e^{i\left(\beta_{i j} k+A_{i, j}\right) x_{j}}}{\sqrt{\beta_{i j} k}} \sum_{j^{\prime}=1}^{N_{V}} a_{i j^{\prime}} \sigma_{j i, i j^{\prime}},
$$

with the appropriate weights $a_{i j^{\prime}}$ corresponding to the incoming flux on the bond $B_{j^{\prime} i}$ towards the vertex $V_{i}$. Comparing this expression to (7.3) yields

$$
b_{i j}=\sum_{j^{\prime}=1}^{N_{V}} \sigma_{j i, i j^{\prime}} a_{i j^{\prime}}
$$

Substituting (7.5) into the boundary conditions (7.1) and (7.2) at the vertex $V_{i}$ we get correspondingly

$$
\sum_{j^{\prime}=1}^{N_{V}} \frac{a_{i j^{\prime}}}{\sqrt{\beta_{i j} k}}\left(\delta_{j j^{\prime}}+\sigma_{j i, i j^{\prime}}\right)=\varphi_{i} C_{i j}
$$

and

$$
\sum_{j, j^{\prime}=1}^{N_{V}} C_{i j} a_{i j^{\prime}} \sqrt{\beta_{i j} k}\left(\delta_{j j^{\prime}}-\sigma_{j i, i j^{\prime}}\right)=i \lambda_{i} \varphi_{i}
$$


Substituting (7.7) into (7.8) one gets

$$
C_{i j} \sum_{l, j^{\prime}=1}^{N_{V}} C_{i l} a_{i j^{\prime}} \sqrt{\beta_{i l} k}\left(\delta_{l j^{\prime}}-\sigma_{l, j^{\prime}}^{(i)}\right)=i \lambda_{i} \sum_{j^{\prime}=1}^{N_{V}} \frac{a_{i j^{\prime}}}{\sqrt{\beta_{i j} k}}\left(\delta_{j j^{\prime}}+\sigma_{j i, i j^{\prime}}\right)
$$

In case of the linear scaling, $\lambda_{i}=k \lambda_{i}^{0}$, this yields

$$
\sum_{j^{\prime}=1}^{N_{V}} a_{i j^{\prime}} C_{i j} \sum_{l=1}^{N_{V}} C_{i l} \sqrt{\beta_{i l}}\left(\delta_{l j^{\prime}}-\sigma_{l i, i j^{\prime}}\right)=i \lambda_{i}^{0} \sum_{j^{\prime}=1}^{N_{V}} \frac{a_{i j^{\prime}}}{\sqrt{\beta_{i j}}}\left(\delta_{j j^{\prime}}+\sigma_{j i, i j^{\prime}}\right)
$$

Comparing the coefficients in front of $a_{i j^{\prime}}$, we get

$$
C_{i j} \sum_{l=1}^{N_{V}} C_{i l} \delta_{l j^{\prime}} \sqrt{\beta_{i l}}-C_{i j} \sum_{l=1}^{N_{V}} C_{i l} \sqrt{\beta_{i l}} \sigma_{l, j^{\prime}}^{(i)}-i \lambda_{i}^{0} \frac{\delta_{j j^{\prime}}}{\sqrt{\beta_{i j}}}=\frac{i \lambda_{i}^{0}}{\sqrt{\beta_{i j}}} \sigma_{j i, i j^{\prime}}
$$

or, after performing the summation over $l$,

$$
C_{i j} C_{i j^{\prime}} \sqrt{\beta_{i j^{\prime}}}-C_{i j} \Gamma_{i, j^{\prime}}^{i}-i \lambda_{i}^{0} \frac{\delta_{j j^{\prime}}}{\sqrt{\beta_{i j}}}=\frac{i \lambda_{i}^{0}}{\sqrt{\beta_{i j}}} \sigma_{j i, i j^{\prime}}
$$

where $\Gamma_{i, j^{\prime}}^{i}=\sum_{l=1}^{N_{V}} C_{i l} \sqrt{\beta_{i l}} \sigma_{l i, i j^{\prime}}$.

Multiplying both sides by $C_{i j} \beta_{i j}$ and summing over $j$ yields

$$
v_{i} C_{i j^{\prime}} \sqrt{\beta_{i j^{\prime}}}-v_{i} \Gamma_{i, j^{\prime}}^{i}-i \lambda_{i}^{0} C_{i j^{\prime}} \sqrt{\beta_{i j^{\prime}}}=i \lambda_{i}^{0} \Gamma_{i, j^{\prime}}^{i}
$$

where $v_{i}=\sum_{j} C_{i j} \beta_{i j}$. Hence

$$
\frac{v_{i}-i \lambda_{i}^{0}}{v_{i}+i \lambda_{i}^{0}} C_{i j^{\prime}} \sqrt{\beta_{i j^{\prime}}}=\Gamma_{i, j^{\prime}}^{i}
$$

which can be used in (7.12) to obtain

$$
C_{i j} C_{i j^{\prime}} \sqrt{\beta_{i j^{\prime}}}-C_{i j} \frac{v_{i}-i \lambda_{i}^{0}}{v_{i}+i \lambda_{i}^{0}} C_{i j^{\prime}} \sqrt{\beta_{i j^{\prime}}}-i \lambda_{i}^{0} \frac{\delta_{j j^{\prime}}}{\sqrt{\beta_{i j}}}=\frac{i \lambda_{i}^{0}}{\sqrt{\beta_{i j}}} \sigma_{j i, i j^{\prime}}
$$

or

$$
\sigma_{j i, i j^{\prime}}=\left(-\delta_{j j^{\prime}}+\frac{2 \sqrt{\beta_{i j} \beta_{i j^{\prime}}}}{v_{i}+i \lambda_{i}^{0}}\right) C_{j i} C_{i j^{\prime}}
$$

We see that in the scaling case the matrix elements $\sigma_{j i, i j^{\prime}}$ of the vertex scattering matrix $\sigma$ are $k$-independent constants. 
The matrix element $\sigma_{j i, i j}$ has the meaning of the reflection coefficient from the vertex $V_{i}$ along the bond $B_{i j}$, and the elements $\sigma_{j i, i j^{\prime}}, j \neq j^{\prime}$ are the transmission coefficients for transitions between different bonds. The equation (7.6) can be written as

$$
\vec{b}=\tilde{T} \vec{a}
$$

where

$$
\tilde{T} \equiv \tilde{T}_{i j, n m}=\delta_{i n} C_{j i} C_{n m} \sigma_{j i, i m}
$$

In the symmetrical basis $\psi_{j i}\left(L_{i j}-x\right)=\psi_{i j}(x)$ one has

$$
\psi_{j i}\left(L_{i j}-x\right)=a_{j i} \frac{e^{i\left(-\beta_{i j} k+A_{j i}\right)\left(L_{i j}-x\right)}}{\sqrt{\beta_{i j} k}}+b_{j i} \frac{e^{i\left(\beta_{i j} k+A_{j i}\right)\left(L_{i j}-x\right)}}{\sqrt{\beta_{i j} k}}=\psi_{i j}(x),
$$

so the coefficients $a_{i j}$ and $b_{i j}$ are related as

$$
a_{j i}=b_{i j} e^{i\left(\beta_{i j} k+A_{i j}\right) L_{i j}}, \quad b_{j i}=a_{i j} e^{i\left(-\beta_{i j} k+A_{i j}\right) L_{i j}}
$$

The coefficients $a_{i j}$ and $a_{j i},\left(b_{i j}\right.$ and $\left.b_{j i}\right)$ are considered different, so the bonds of the graph are "directed".

Equations (7.20) can be written in matrix form,

$$
\vec{a}=P \tilde{D}(k) \vec{b}
$$

where $\vec{a}$ and $\vec{b}$ are the $2 N_{B}$ dimensional vectors of coefficients and $\tilde{D}$ is a diagonal matrix in the $2 N_{B} \times 2 N_{B}$ space of directed bonds,

$$
\tilde{D}_{i j, p q}(k)=\delta_{i p} \delta_{j q} e^{i\left(\beta_{i j} k+A_{i j}\right) L_{i j}}
$$

and

$$
P=\left(\begin{array}{cc}
0 & 1_{N_{B}} \\
1_{N_{B}} & 0
\end{array}\right)
$$

where $1_{N_{B}}$ is the $N_{B}$-dimensional unit matrix. The pairs of indices $(i j),(p q)$, identifying the bonds of the graph $\Gamma$, play the role of the indices of the matrix $\tilde{D}(k)$. 
Equations (7.21) and (7.17) together result in

$$
\vec{a}=S(k) \vec{a},
$$

with the matrix $S(k)$ (the total graph scattering matrix) given by

$$
S(k)=D(k) T,
$$

where $D=P \tilde{D} P$ and $T=P \tilde{T}$. 


\section{REFERENCES}

[1] L. D. Landau and E. M. Lifshitz, Quantum Mechanics (Pergamon Press, Oxford, New York, 1977).

[2] A. Messiah, Quantum Mechanics (North-Holland, Amsterdam, 1961).

[3] S. Flügge, Practical Quantum Mechanics (Springer, Berlin, 1999).

[4] R. Blümel, Yu. Dabaghian, and R. V. Jensen, Phys. Rev. Lett., in press.

[5] Yu. Dabaghian, R. V. Jensen, and R. Blümel, Pis'ma v ZhETF 74, 258 (2001); JETP Lett. 74, 235 (2001).

[6] R. Blümel, Yu. Dabaghian, and R. V. Jensen, submitted to Phys. Rev. E.

[7] H. Bohr, Almost Periodic Functions (Chelsea Publishing, New York, 1951).

[8] H. Friedrich, Theoretical Atomic Physics (Springer, Berlin, 1990).

[9] H.-J. Stöckmann, Quantum Chaos (Cambridge University Press, Cambridge, 1999).

[10] R. Blümel, Yu. Dabaghian, and R. V. Jensen, Mathematical Foundations of Regular Quantum Graphs, in preparation.

[11] M. Gutzwiller, Chaos in Classical and Quantum Mechanics (Springer, New York, 1990).

[12] J. B. Keller, Ann. Phys. (N.Y.) 4, 180 (1958).

[13] R. I. Szabo, Equivariant Localization of Path Integrals (LANL archive hep-th/9608068, 1996).

[14] M. V. Berry, "Quantum chaology: our Knowledge and Ignorance", in New Trends in Nuclear Collective Dynamics, Springer Proceedings in Physics, Vol. 58, eds. Y. Abe, H. Huriuchi, K. Matsuyanagi, pp. 177-181 (Springer Verlag, Berlin, 1991).

[15] J.-P. Roth, in Lecture Notes in Mathematics: Théorie du Potentiel, Vol. 1096, A. Dold and B. Eckmann, eds. (Springer, Berlin, 1984) pp. 521-539. 
[16] T. Kottos and U. Smilansky, Phys. Rev. Lett. 79, 4794 (1997); Ann. Phys. (N.Y.) 274, 76 (1999).

[17] M. Pascaud and G. Montambaux, Phys. Rev. Lett. 82, 4512 (1999).

[18] H. Schanz and U. Smilansky, Phys. Rev. Lett. 84, 1427 (2000).

[19] Y. Dabaghian, R. V. Jensen, and R. Blümel, Phys. Rev. E 63, 066201 (2001).

[20] Y. C. Lai, C. Grebogi, R. Blümel, and M. Ding, Phys. Rev. A 45, 8284 (1992).

[21] M. Keeler and T. J. Morgan, Phys. Rev. Lett. 80, 5726 (1998).

[22] V. I. Arnold and A. Avez, Ergodic Problems of Classical Mechanics (Addison Wesley, Redwood City, 1989).

[23] V. I. Arnold, Mathematical Methods of Classical Mechanics (Springer, New York, 1997).

[24] E. Akkermans, A. Comtet, J. Desbois, G. Montambaux, and C. Texier, Spectral Determinant on Quantum Graphs (LANL archive cond-mat/9911183, 1999).

[25] F. Barra and P. Gaspard, Phys. Rev. E 63, 066215 (2001).

[26] R. E. Prange, E. Ott, T. M. Antonsen, B. Georgeot, and R. Blümel, Phys. Rev. E 53, 207 (1996).

[27] R. Blümel, T. M. Antonsen, Jr., B. Georgeot, E. Ott, and R. E. Prange, Phys. Rev. Lett. 76, 2476 (1996); Phys. Rev. E 53, 3284 (1996).

[28] L. Sirko, P. M. Koch, and R. Blümel, Phys. Rev. Lett. 78, 2940 (1997).

[29] U. Smilansky, in Chaos et Physique Quantique-Chaos and Quantum Physics, Les Houches session LII, 1989, M.-J. Giannoni, A. Voros, and J. Zinn-Justin, eds. (Elsevier Science Publishers, Amsterdam, 1991), pp. 371-441.

[30] R. Courant and D. Hilbert, Methods of mathematical physics (New York, Interscience Publishers, 1953). 
[31] R. Blümel and Yu. Dabaghian, J. Math. Phys, in press (2001).

[32] A. Selberg, J. Indian Math. Soc. B 20, 47 (1956); reprinted in Atle Selberg: Collected Works, Vol. 1 (Springer, Berlin, 1989), pp. 423-463.

[33] K. G. Anderson and R. B. Melrose, Inv. Math. 41, 197 (1977).

[34] H. P. Baltes and E. R. Hilf, Spectra of Finite Systems (Bibliographisches Institut AG, Zürich, 1976). 


\section{Figure Captions}

Fig. 1: Sketch of a step-potential in a box, a well-known textbook quantum problem.

Fig. 2: Sample graph with five vertices and seven bonds.

Fig. 3: The exact spectral staircase $N(k)$ and its average $\bar{N}(k)$ for the scaling step-potential shown in Fig. 1 with $b=0.3$ and $\lambda=1 / 2$. The average $\bar{N}(k)$ crosses every "stair" of $N(k)$ (piercing average) at the equally spaced separating points $\hat{k}_{n}$.

Fig. 4: Collection of potentials and their associated linear quantum graphs that serve as examples to illustrate the concept of regular quantum graphs. (a) Scaling step-potential in a box and its associated three-vertex linear graph (a'). (b) Scaling $\delta$-function in a box and its corresponding three-vertex linear graph (b'). Combined scaling $\delta$-function and step potential in a box (c) with its linear three-vertex quantum graph (c'). Two scaling steps (d) and two scaling $\delta$-functions (e) in a box together with their associated four-vertex dressed linear quantum graphs (d') and (e'), respectively.

Fig. 5: Comparison between the exact eigenvalues $k_{n}$ and the $k_{n}$-values computed via (4.3) for the scaling step-potential shown in Fig. 1. Shown is the relative error $\epsilon_{n}^{(l)}=\left|k_{n}^{(l)}-k_{n}\right| / k_{n}$, $n=1,10,100$, of the result $k_{n}^{(l)}$ predicted by (4.3) compared to the numerically obtained exact result $k_{n}$ as a function of the binary code length $l$ of the orbits used in the expansion (4.3). We used $b=0.3, \lambda=1 / 2$.

Fig. 6: Four-vertex linear chain graph (a) and the corresponding space $\left(r_{2}, r_{3}\right)$ of reflection coefficients (b). The shaded region in the $\left(r_{2}, r_{3}\right)$ space corresponds to the regular regime of the quantum graph shown in (a). This demonstrates that the subset of regular quantum graphs within the set of all four-vertex linear quantum graphs is non-empty and of finite measure.

Fig. 7: The exact spectral staircase $N(k)$ and its average $\bar{N}(k)$ for the regular $r_{2}=0.2$, $r_{3}=0.3$ ) (a) and the irregular $\left.r_{2}=0.98, r_{3}=0.99\right)(\mathrm{b})$ regimes of the four-vertex linear 
graph shown in Fig. 6a. In the regular regime (a) the average staircase $\bar{N}(k)$ pierces every step of $N(k)$. This is not the case in (b), characteristic of the irregular regime.

Fig. 8: Two-vertex circular graph. In the mathematically positive sense, $\beta_{12}$ is the scaling coefficient of the bond connecting vertex $V_{1}$ with vertex $V_{2}, \beta_{21}$ is the scaling coefficient of the bond connecting $V_{2}$ with $V_{1}$. This labelling is possible only in the absence of a magnetic field $\left(A_{i j}=0\right)$ where the sense of traversal of a bond is irrelevant.

Fig. 9: Scaling star-graph with three bonds and four vertices.

Fig. 10: Sketch of a piecewise constant potential ("Manhattan potential") (a) and its associated linear graph (b). 


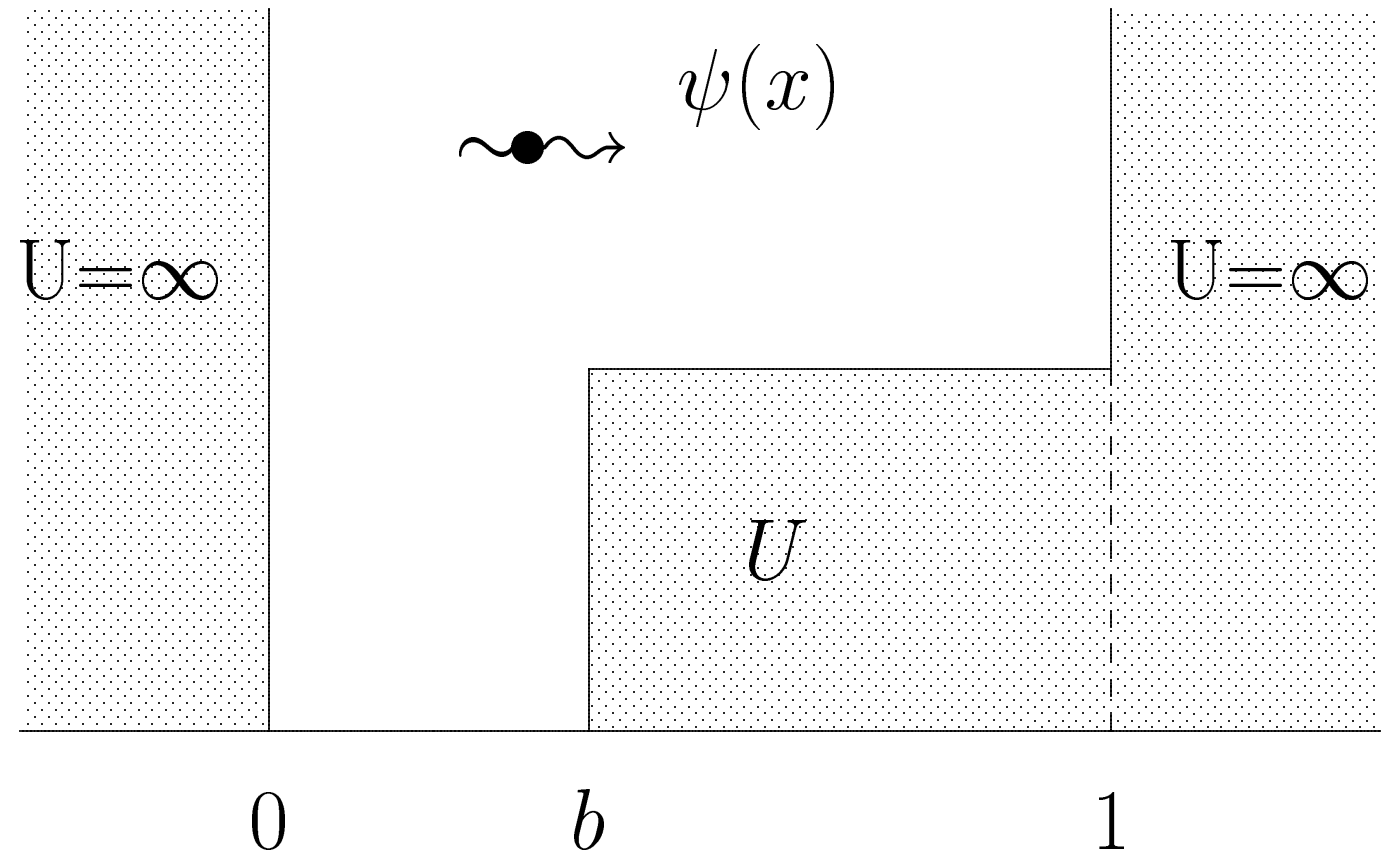

Fig. 1 


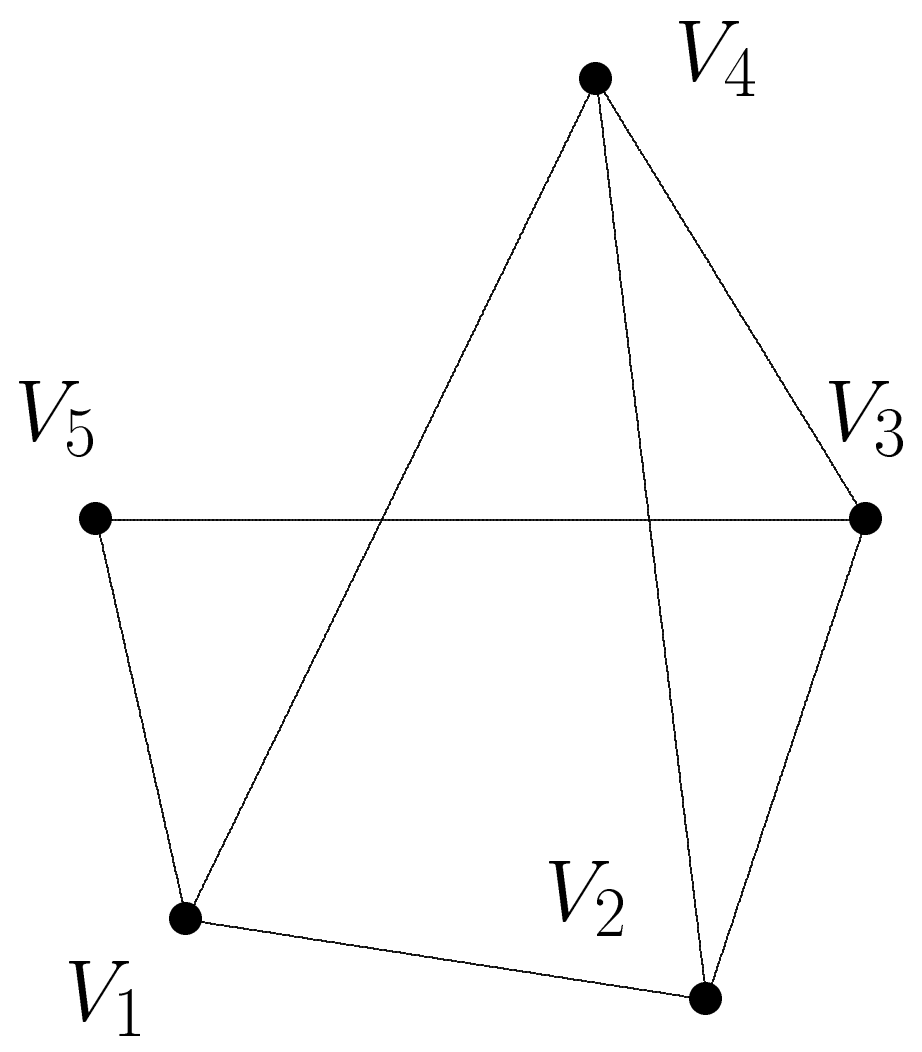

Figure 2 


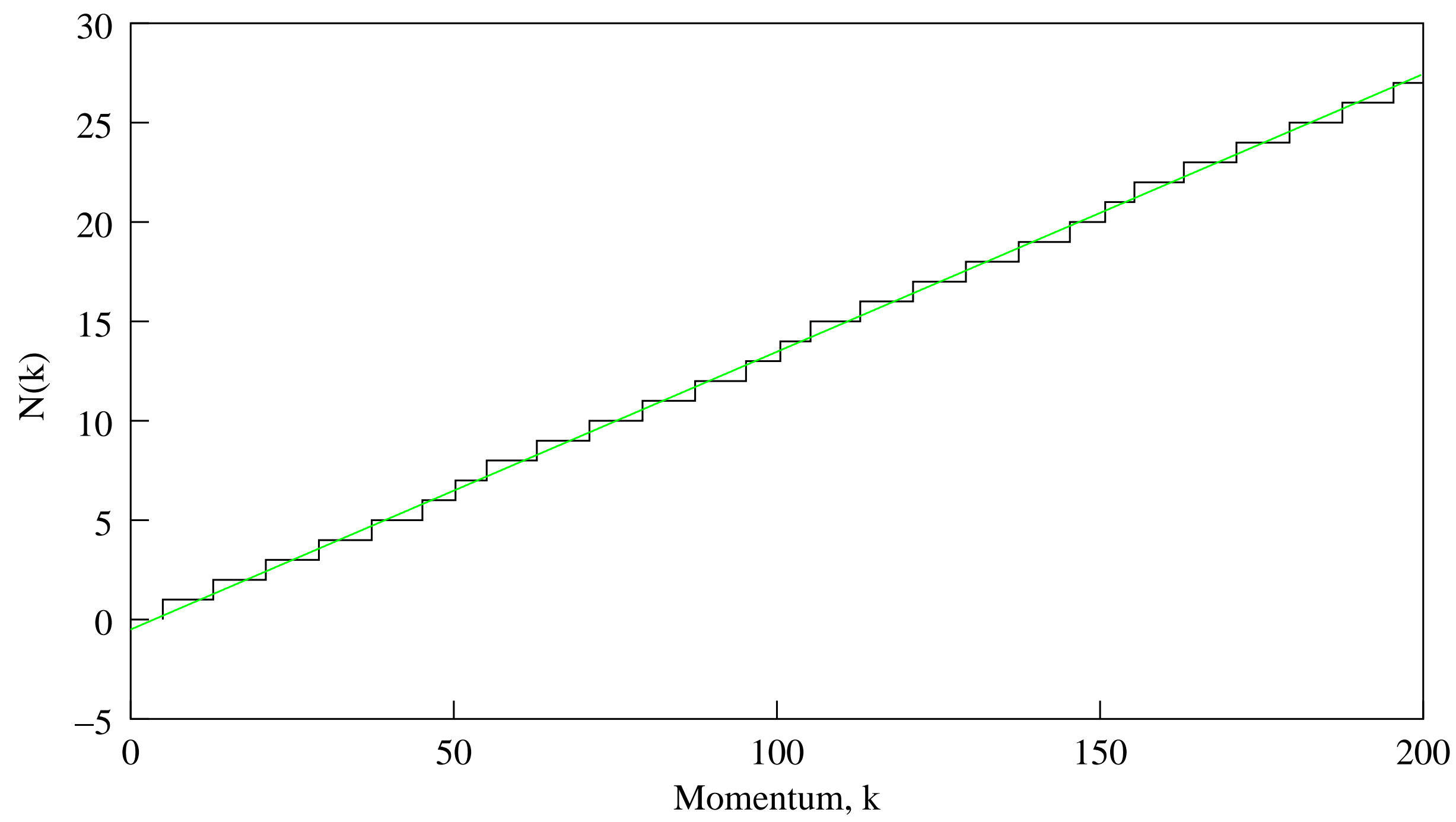

Fig. 3 

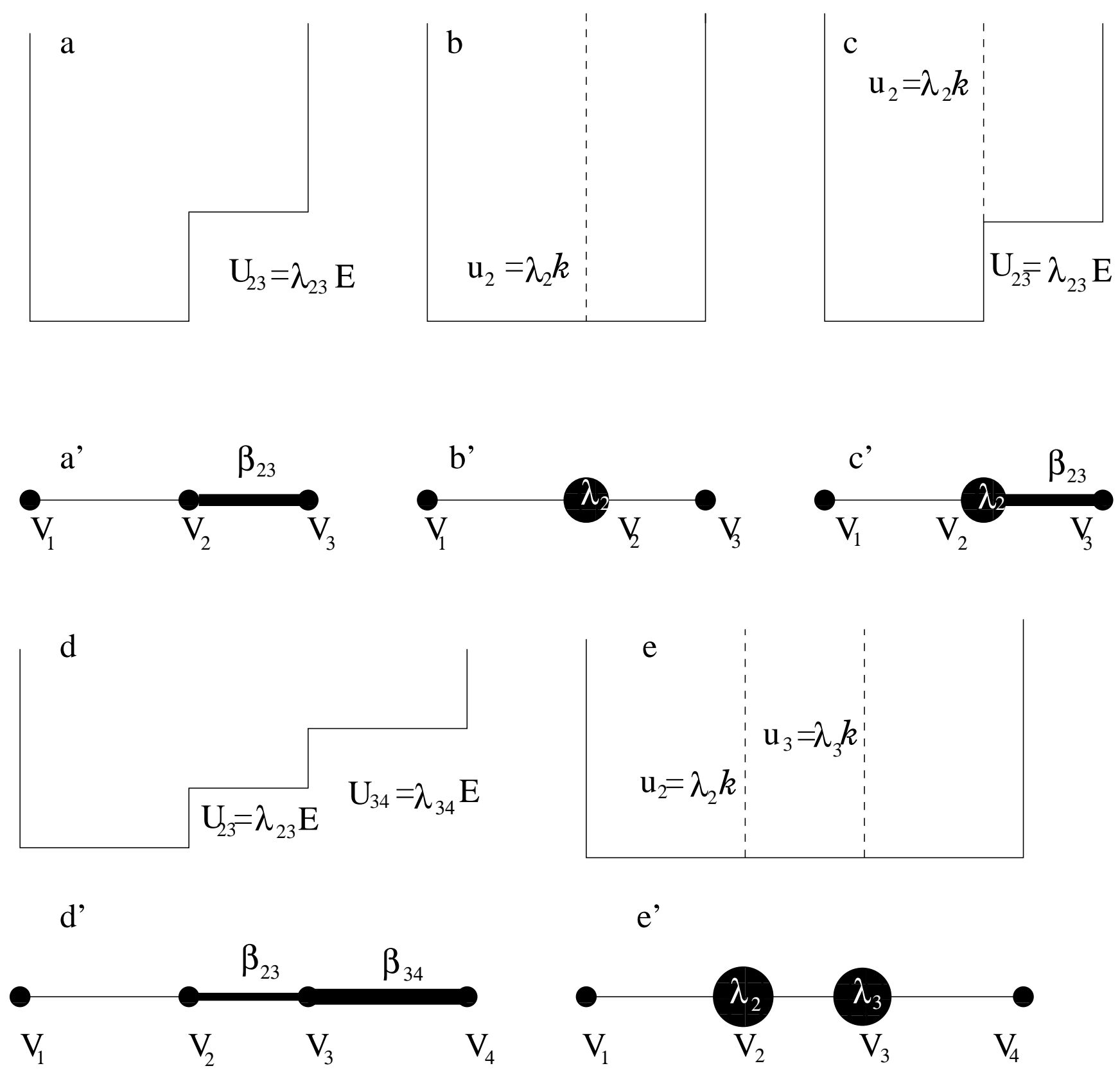

Fig. 4 


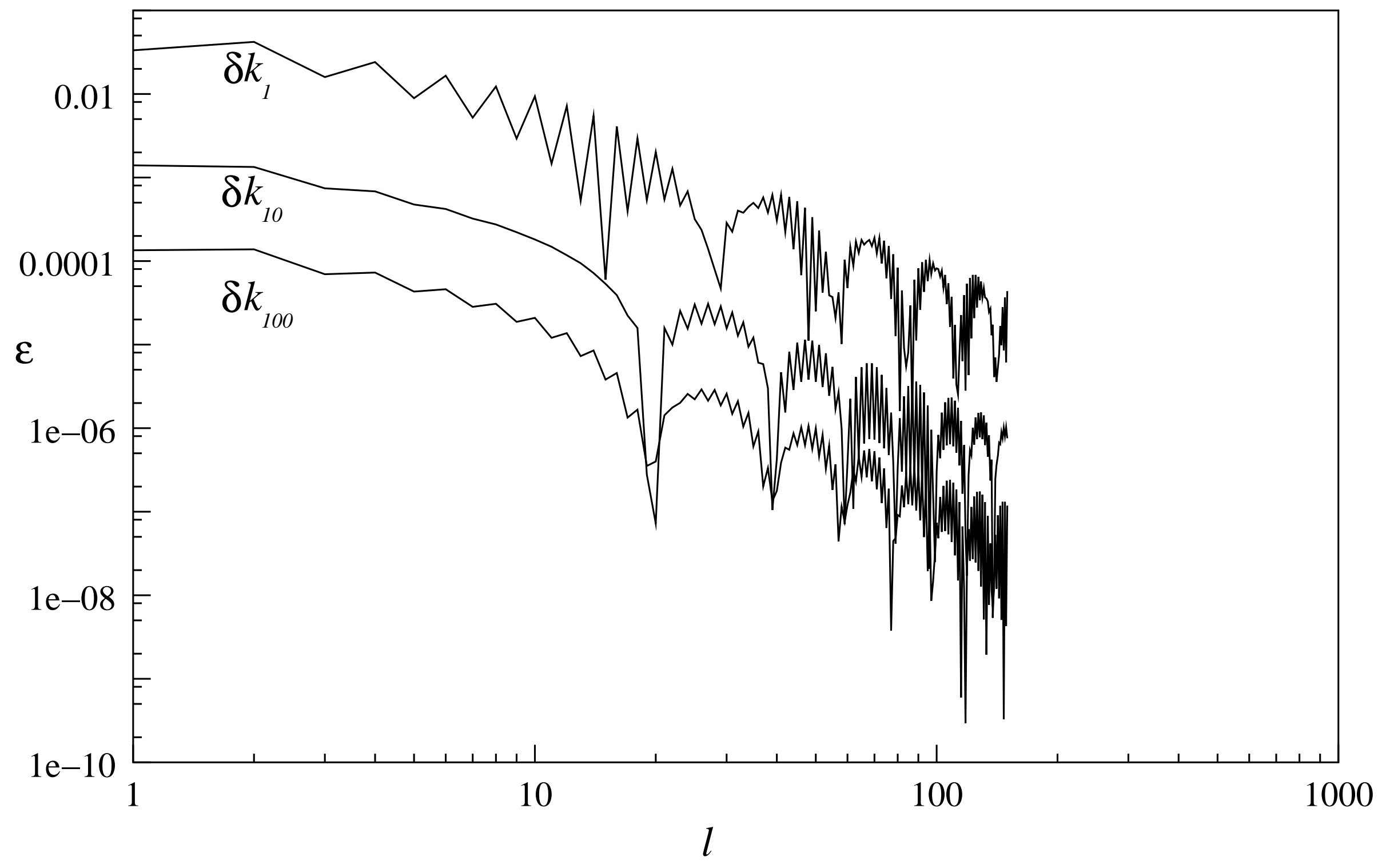

Fig. 5 

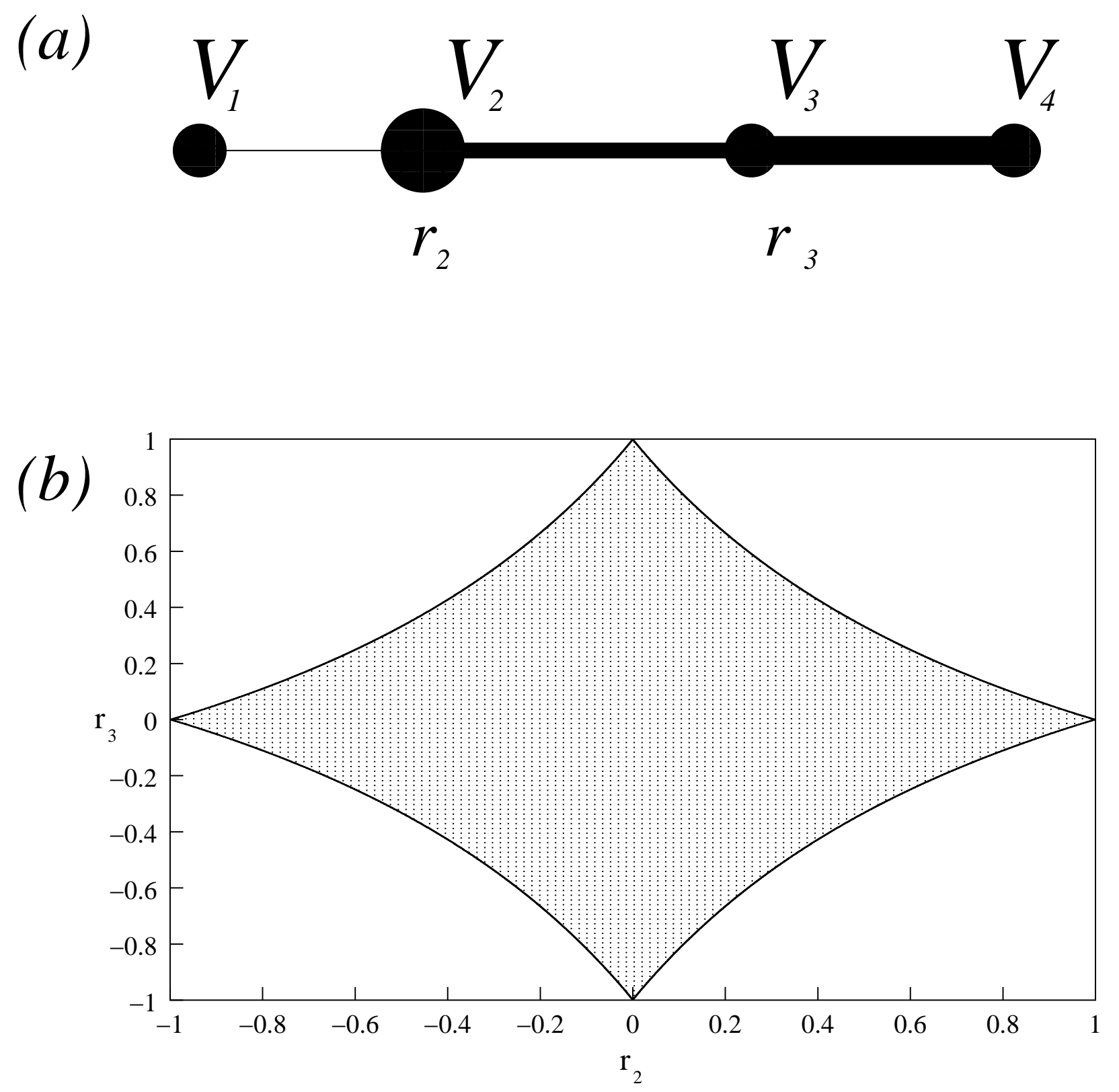

Fig. 6 

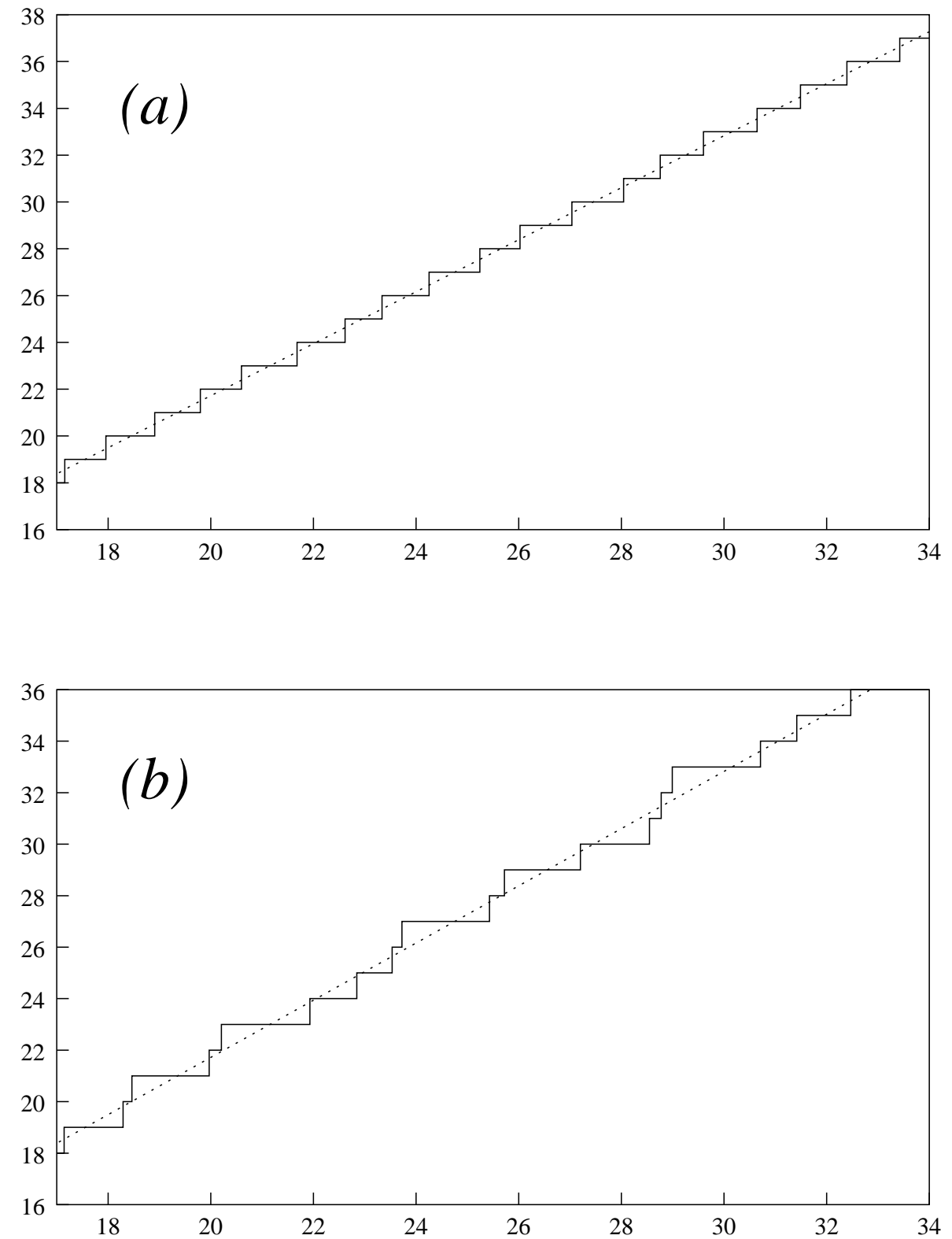

Fig. 7 


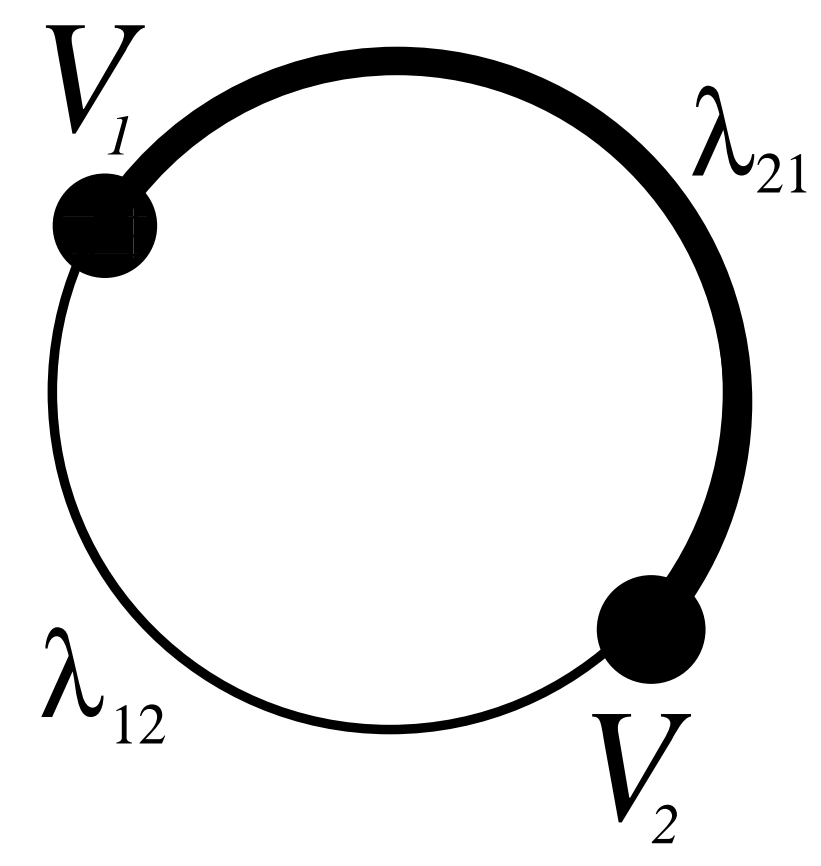

Fig. 8 


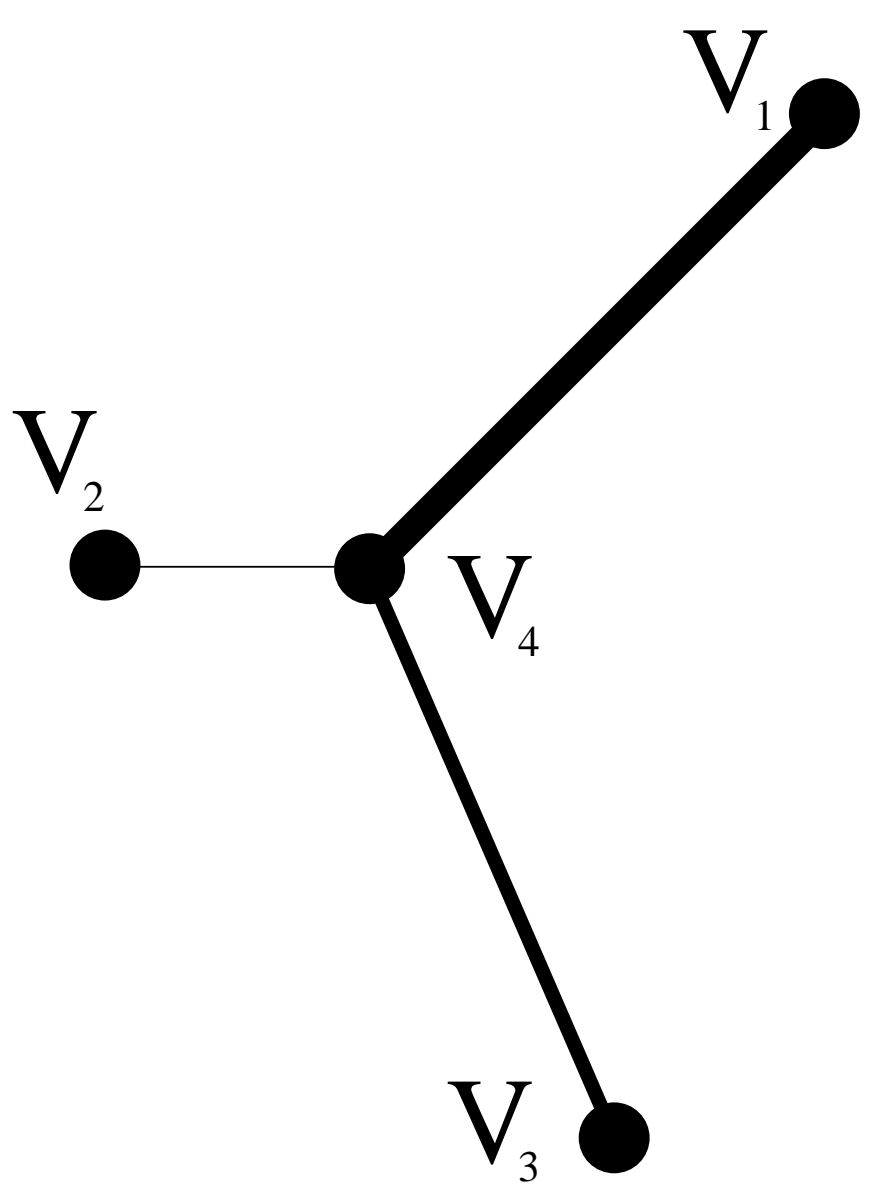

Fig. 9 


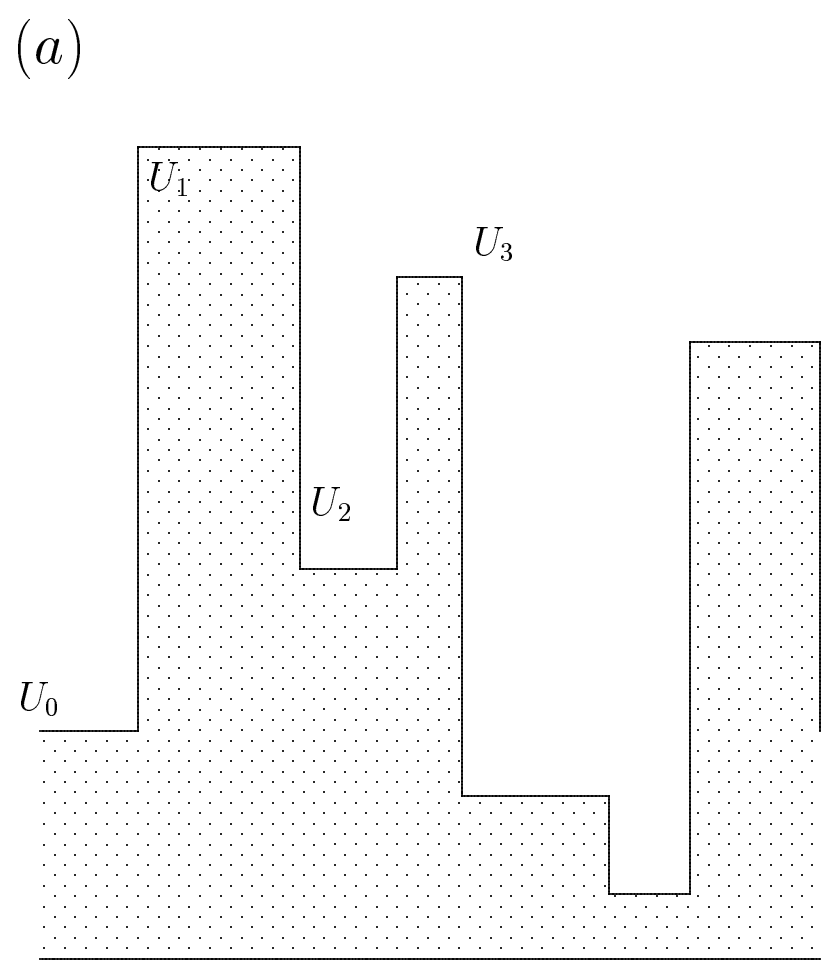

$a_{0} \quad a_{1}$

$a_{2} \quad a_{3} \quad a_{4}$

$a_{5}$

(b)

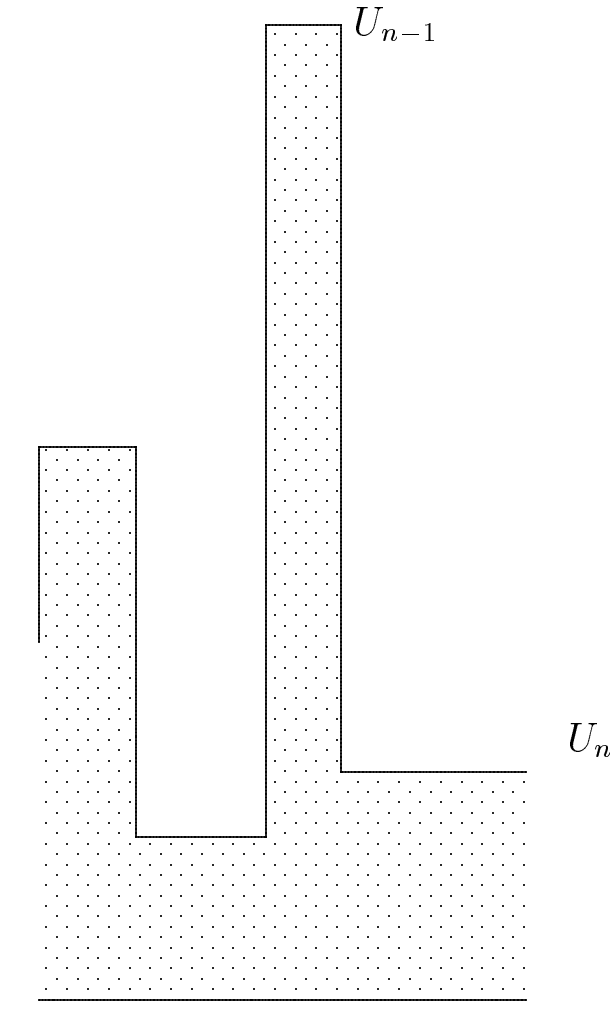

$a_{n-1} \quad a_{n}$

Fig. 10 